\title{
TEXISTEPEC POPOLUCA
}

\section{Søren Wichmann, Lynda Boudreault ${ }^{1}$}

Texistepec Popoluca, a Gulf Zoquean language, is spoken in the town of Texistepec, located in the southernmost part of the state of Veracruz, Mexico. It is one of the four languages spoken in Veracruz that is referred to as Popoluca. Some scholars prefer to call it Texistepequeño and the autodenomination is wi:-Po:t 'good word(s)'.

The story presented here was narrated in 1993 by Tomás Lopéz Florentino. This story was originally published in Cuentos y colorados en popoluca de Texistepec (Wichmann 1996), a collection of Texistepec stories with Spanish translations, an analytical index, and folkloristic commentary. At the time this story was recorded there were at most a few hundred speakers of the language. The stories were recorded on cassette and later transcribed and translated with the help of Mr. López, and to a lesser extent with the late Carmen Román Telésforo.

The representation of the person marking paradigm used in this text differs from the other texts in this volume as there are two intransitive paradigms reflected in the notation. $S_{A}$ marks ergative subjects of intransitive verbs, and $\mathrm{S}_{\mathrm{B}}$ marks absolutive subjects of intransitive verbs. Additionally, in this text the symbol ê represents an underlying segment that surfaces as [e], but which is subject to allophonic variation that distinguishes it from /e/. It is not in itself a phoneme; the use of the symbol here is strictly analytical.

\section{KAÑCHEÑYOOMÄ 'THE LAZY WOMAN'}

\section{TomÁs LopÉz Florentino}

The story Kañcheñyoomä' 'The Lazy Woman' (kañčeñ 'lazy', yo:mit? 'woman') is told in a series of episodes. It tells of a young man who wants to take a wife, but the object of his affection is known to be lazy (lines 1-60). After they are married, his bride's lack of interest in helping with household chores frustrates her new family, especially her mother-in-law (61-93). After the couple is moved into their own home, the young man learns the extent of her laziness (104-154) and solves the problem (155-200). His fatherin-law sees first-hand how his daughter has changed (201-244) and learns what motivated her transformation (245-283). The father-in-law returns home with notions of implementing the same strategy to change his wife's lifelong

${ }^{1}$ Wichmann recorded and analyzed the text; Boudrealt prepared the English translation and did the formatting; Jan Strunk is responsible for text-to-speech alignment.

[IJAL, vol. 83, Supp. 1, TILA 1, January 2017, pp. S57-S102]

(C) 2017 by The University of Chicago. All rights reserved.

0020-7071/2017/83S1-0004\$10.00 
attitude toward domestic responsibility (285-305). His actions have consequences (306-363), and his wife sets him straight (364-395).

(1) Yä'äp tum pääñ.

yịip tum pi:ñ

this one man

'This was a man.'

(2) Tum suutu' pwe.

tum su:tu? pues

one boy well

'Well, a boy.'

(3) Ma' yakkä' ’komtajam.

ma? $\emptyset$-yak-kînkom-tah=am

PERF $3 \mathrm{~S}_{\mathrm{B}}$-CAUS-marry-PASS=IAM

'They married.'

(4) Ma' dyyakkä'Đkom yoom je'ba'a tum yoomä',

ma? y-yak-kînkom y-?o:m he?-ba?a tum yo:mi?

PERF 3A:3O-CAUS-marry 3PSR-father 3PRO-with one woman

'His father married him to a woman,'

(5) Pero njumbu' kañcheñ 'yaap,

pero nhumbu? $\emptyset$-kañčeñ $\quad y-3 a: p$

but very 3S -lazy 3PSR-mother

'But her mother was very lazy,'

(6) I njumbu' kañcheñ nee dyyombaa.

?i nhumbu? $\emptyset$-kañčeñ ne: y-yo:mi?-ba:

and very 3S $\mathrm{S}_{\mathrm{B}}$-lazy also 3PSR-woman-DIM

'And her daughter was very lazy too.'

(7) I jeksh boy wya'k.

?i hekš boy y-wa?k

and now go.and.return ${ }_{\mathrm{AUX}}$ :PERF 3A:3O-ask

'And now he went to ask (her father).'

(8) Dyim, Papa dyim, n'oom, eñch n'eshp a'y däk knwya'ka' dyim, nuuñe'.

y-dim papá $y-\operatorname{dim} \quad n-$-To:m Reñč n-?êš-p

$3 \mathrm{~S}_{\mathrm{A}}$-say father $3 \mathrm{~S}_{\mathrm{A}}$-say 1PSR-father NEG 1A:3O-know-FUT

?a?y dik kny-wa?k-a? y-dim n-du:ñe?

if $\quad \mathrm{go}_{\mathrm{AUX}} \quad 2 \mathrm{~A}: 1 \mathrm{O}-\mathrm{ask}-\mathrm{APPL} \quad 3 \mathrm{~S}_{\mathrm{A}}$-say $1 \mathrm{PSR}$-woman

'He says, "Papa, I don't know if you can ask (for)," he says, "my wife.", 
(9) Si, däk ta nwa'k dyim.

$\begin{array}{lllll}\text { sí } & \text { dik } & \text { ta } & \text { n-wa?k } & \text { y-dim } \\ \text { yes } & \text { go }_{\mathrm{AUX}} & \text { INCL } & 1 \mathrm{~A}: 3 \mathrm{O}-\mathrm{ask} & 3 \mathrm{~S}_{\mathrm{A}}-\text { say }\end{array}$

“"Yes, let's go ask," (his father) says.'

(10) Ee dyyombaa?

?e: y-yo:mir-ba:

who 3PSR-woman-DIM

" "Whose daughter is she?",

(11) Julaanu, dyim,

Julano y-dim

Fulano $3 \mathrm{~S}_{\mathrm{A}}-$ say

“"Fulano," he says.' 2

(12) No dyim, chi' nwyata'ap jepe' nje' dyim.

no $y$-dim či? ny-wat-a?-p hepe? nhe?

no $3 \mathrm{~S}_{\mathrm{A}}-$ say what $2 \mathrm{~A}: 3 \mathrm{O}-$ do-APPL-FUT this $3 \mathrm{PRO}$

$$
\mathrm{y}-\operatorname{dim}
$$

$3 \mathrm{~S}_{\mathrm{A}}-\mathrm{say}$

“"No," he says, "Why do you want her? No.'

(13) Uu! dyim, njumbu? kañcheñ dyim.

$\mathrm{u}: \quad \mathrm{y}-\mathrm{dim}$ nhumbu? $\emptyset$-kañčeñ $\mathrm{y}-\mathrm{dim}$

INTER $3 \mathrm{~S}_{\mathrm{A}}$-say very $3 \mathrm{~S}_{\mathrm{B}}$-lazy $3 \mathrm{~S}_{\mathrm{A}}-$ say

“"Oh! She's very lazy.'

(14) Njumbu'kañcheñ, dyim.

nhumbu? $\emptyset$-kañčeñ $\quad \mathrm{y}$-dim

very $\quad 3 \mathrm{~S}_{\mathrm{B}}-$ lazy $3 \mathrm{~S}_{\mathrm{A}}-$ say

“"She's very lazy," he says.'

(15) Eñch shyun yoswatp.

?eñč y-sun $\emptyset$-yo:s-wat-p

no 3A:3O-want $3 \mathrm{~S}_{\mathrm{B}}$-work-do-FUT

“"She doesn't want to work.'

(16) Chi' nwyata'ap? Meñche nchyuunpakp!

či? ny-wat-a?-p mêñ-če ny-tu:n-pak-pe

why 2A:3O-do-APPL-FUT come $_{\text {AUX }}$-LIM 2A:3O-sit-have-FUT

“"Why do you want her? You're going to have her sitting!"

(17) No dyim, eñdye nsun mej yä'ä.

no $y$-dim ?eñdye n-sun $\emptyset$-meh yi?i

no $3 \mathrm{~S}_{\mathrm{A}}$-say NEG 1A:30-want $3 \mathrm{~S}_{\mathrm{B}}$-come:Fut here

" "No," he says, "I don't want her to come here.'

2 The name Fulano (of which Julano is a local variant) is frequently used in Spanish to mean 'whoever' or to refer to a nameless someone. 
(18) U nsun ñyuuñe' dyim, meñpä' kki'pa't sikyeera dyim.

\begin{tabular}{|c|c|c|c|c|}
\hline Pu & n-sun & ny-du:ñe? & $\mathrm{y}-\operatorname{dim}$ & $\emptyset-\mathrm{mê} \tilde{n}=\mathrm{pi} ?$ \\
\hline IMPF & 1A:30-want & 2PSR-woman & $3 \mathrm{~S}_{\mathrm{A}}-$ say & $3 \mathrm{~S}_{\mathrm{B}}-$ come $_{\mathrm{AUX}}=\mathrm{REL}$ \\
\hline & $\begin{array}{l}\text {-ki?-pa?t } \\
\text {-hand-find }\end{array}$ & $\begin{array}{ll}\text { siquiera } & \mathrm{y}-\mathrm{dim} \\
\text { at.least } & 3 \mathrm{~S}_{\mathrm{A}}-\mathrm{sa}\end{array}$ & & \\
\hline
\end{tabular}

“"We would want your wife to come to help you at least," he says.'

(19) Kki'pa't juch kdyyoswatp dyim.

ky-kì-pait huč ky-yo:s-wat-p y-dim

3/2-hand-find where $2 \mathrm{~S}_{\mathrm{B}}$-work-do-Fut $3 \mathrm{~S}_{\mathrm{A}}-$ say

“"She's going to help you where you work.'

(20) Byumbe jes kbij, byumbe shyun bich ñiiywatp.

y-bumbe hes ky-bêh

$3 \mathrm{~S}_{\mathrm{A}}-$ all when $2 \mathrm{~S}_{\mathrm{B}}$-come:FUT

$$
\begin{array}{llll}
\text { y-bumbe } & \text { y-sun } & \text { bič } & \text { ny-di:y-wat-p } \\
3 \mathrm{~S}_{\mathrm{A}} \text {-all } & \text { 3A:3O-want } & \text { 2PRO } & \text { 2A:3O-self-do-FUT }
\end{array}
$$

“"When you come, she's going to want you to do everything yourself.",

(21) Pero ätz nwä'yjaam.

pero iic n-wi?nha:m

but 1PRO 1A:3O-like

، "But I like her.'

(22) Ätz u nsun.

Pic $\mathrm{Pu}$ n-sun

1PRO IMPF 1A:3O-want

" "I want her.",

(23) Bich njyaam, maan,

bič ny-ha:m n-ba:n

2PRO 2A:3O-know 1PSR-child

" "You'll find out, son,'

(24) Ätz ma'ye knäma', jepe' yoomä' ma' matän ke njumbu'm kañcheñ. iic ma?=ye kn-dim-a? hepe? yo:mi? ma? 1PRO PERF=already 1A:2O-say-APPL this woman PERF

$$
\begin{aligned}
& \text { n-batin que nhumbu?=m } \emptyset \text {-kañčeñ } \\
& \text { 1A:3O-hear that very=REP } 3 \mathrm{~S}_{\mathrm{B}} \text {-lazy }
\end{aligned}
$$

“"(but) I tell you, I've heard this girl is really lazy.",

(25) Aa, no le hace.

'Ah, it doesn't matter to him.' 
(26) Pwe, ätz u nsun.

pues ?iф ?u n-sun

well 1PRO IMPF 1A:3O-want

“"Well, I want her.",

(27) Bweenu, bich njyaam.

bueno bič ny-ha:m

good 2PRO 2A:3O-know

“'Well, you know (best).'

(28) Ätz däk ta nwa'k shaaptu.

?i: $\varnothing$ dik ta n-wa?k ša:ptu

1 PRO $\mathrm{go}_{\mathrm{AUX}}$ INCL 1A:3O-ask Saturday

“"We'll go ask this Saturday.'

(29) Shaaptu däk ta nwa'k.

ša:ptu dik ta n-wa?k

Saturday $\mathrm{go}_{\mathrm{AUX}}$ INCL $1 \mathrm{~A}: 3 \mathrm{O}-$ ask

"'Saturday we'll go ask.",

(30) Pwe ta däkp dyim.

pues ta dik-p y-dim

well INCL go-FUT $3 \mathrm{~S}_{\mathrm{A}}$-say

“"Well, let's go," he says.'

(31) Ma' däkyajam.

ma? $\emptyset$-dik-yah=am

PERF $3 \mathrm{~S}_{\mathrm{B}}-\mathrm{gO}-3 \mathrm{PL}=\mathrm{IAM}$

'They went.'

(32) Ma' du'k shaaptu.

ma? $\emptyset-$ du?k ša:ptu

PERF $3 \mathrm{~S}_{\mathrm{B}}$-arrive Saturday

'Saturday came.'

(33) Ma' däkyaj.

ma? $\emptyset$-dik-yah

PERF $3 \mathrm{~S}_{\mathrm{B}}-\mathrm{gO}-3 \mathrm{PL}$

'They went.'

(34) Boy wya'kyaj jepe' yoomä'.

boy $\quad \mathrm{y}$-wa?k-yah hepe? yo:mi? go.and.return $_{\text {AUX }}:$ PERF $3 \mathrm{~A}: 3 \mathrm{O}-$ ask-3PL this woman

'They went to ask for this woman.' 
(35) Ma' du'kam njem.

ma? $\emptyset$-du?k=am nhem

PERF $3 \mathrm{~S}_{\mathrm{B}}$-arrive $=\mathrm{IAM}$ there

'They arrived there.'

(36) Pero ätzä' ma' kbeñta' dyim, poko yä'äp suutu' dyim, ma' shyun ñyombaa dyim.

pero ?iфi? ma? k-bêñ-ta? y-dim poco yi?ip su:tu? but $1 \mathrm{PRO}$ PERF $1 \mathrm{~S}_{\mathrm{B}}$-come-1PL $3 \mathrm{~S}_{\mathrm{A}}$-say because this boy

$$
\begin{array}{lllll}
\text { y-dim } & \text { ma? } & \text { y-sun } & \text { ny-yo:mir-ba: } & \text { y-dim } \\
\text { 3S } \mathrm{S}_{\mathrm{A}} \text {-say } & \text { PERF } & \text { 3A:3O-want } & \text { 2PSR-woman-DIM } & \text { 3S } \mathrm{S}_{\mathrm{A}} \text {-say }
\end{array}
$$

" "But we have come," he says, "because this boy says he is in love with your daughter," he says.'

(37) Eñch n'esh njune' ma' shyunka'yaj dyim,
?eñč n-?êš
nhune? ma? y-sun-ka?yah
$\mathrm{y}-\mathrm{dim}$
no 1A:30-know how
PERF 3A:3O-want-REC $3 \mathrm{~S}_{\mathrm{A}}$-say

“ "I don't know how they fell in love," he says,'

(38) pero en fin dyim, ma' kbeñ pa nwa'kta'

pero en fin $y$-dim ma? k-bêñ pa n-wa?k-ta?

but in end $3 \mathrm{~S}_{\mathrm{A}}$-say PERF $1 \mathrm{~S}_{\mathrm{B}}$-come for 1A:30-ask-1PL

“"but in the end," he says, "we've come to ask for her,'

(39) i nwa'ktzä'yta' dyim.

?i n-wa?k-ci?y-ta? y-dim

and 1A:3O-ask-stay-1PL $3 \mathrm{~S}_{\mathrm{A}}$-say

“"and we're going to ask," he says.'

(40) Wää dyim.

wi: $\quad y-d i m$

good $3 \mathrm{~S}_{\mathrm{A}}-\mathrm{say}$

، "Good," he says.'

(41) Ma' näm 'yoom,

ma? $\emptyset$-dim y-?o:m

PERF $3 \mathrm{~S}_{\mathrm{B}}$-say 3PSR-father

'Her father said,'

(42) Wää dyim,

$\emptyset$-wi: $\quad$-dim

$3 \mathrm{~S}_{\mathrm{B}}$-fine $3 \mathrm{~S}_{\mathrm{A}}$-say

“"That's fine," he says.'

(43) pero batänä' dyim, knämpaapä' dyim.

pero $\emptyset$-batin-i? $\quad \mathrm{y}$-dim $\quad \mathrm{kn}-\mathrm{dim}-\mathrm{pa}=\mathrm{pir} \quad \mathrm{y}-\mathrm{dim}$

but $3 \mathrm{~S}_{\mathrm{B}}$-hear-IMP $3 \mathrm{~S}_{\mathrm{A}}$-say $1 \mathrm{~A}: 2 \mathrm{O}-\mathrm{say}-\mathrm{NOM}=\mathrm{REL} \quad 3 \mathrm{~S}_{\mathrm{A}}-$ say

“"but listen," he says, "to what I tell you!" he says.' 
(44) Chi'yoo? dyim.

čîyo: $\quad \mathrm{y}-\mathrm{dim}$

what $\quad 3 \mathrm{~S}_{\mathrm{A}}-$ say

" "What?" he says.'

(45) Yä'äpä' nyombaa dyim, eñdyee dyyoswat dyim.

yîipi? n-yo:mi?-ba: y-dim ?eñdye: y-yo:s-wat

this 1PSR-woman-DIM $3 \mathrm{~S}_{\mathrm{A}}$-say $\mathrm{NEG} \quad 3 \mathrm{~S}_{\mathrm{A}}$-work-do

$$
\begin{aligned}
& \mathrm{y}-\mathrm{dim} \\
& 3 \mathrm{~S}_{\mathrm{A}}-\mathrm{say}
\end{aligned}
$$

“" 'My daughter doesn't work.'

(46) Eñdyee dyyoswat dyim,

?eñdye y-yo:s-wat $y-d i m$

NEG $\quad 3 \mathrm{~S}_{\mathrm{A}}$-work-do $3 \mathrm{~S}_{\mathrm{A}}-$ say

“"She doesn't work," he says.'

(47) Yä'äpä' eñdye dyyoswat dyim.

yi̊ipi? ?eñdye y-yo:s-wat y-dim

this NEG $\quad 3 \mathrm{~S}_{\mathrm{A}}$-work-do $3 \mathrm{~S}_{\mathrm{A}}-$ say

“"She doesn't work," he says.'

(48) I asi es ke ätz eñch n'eshp a'y nwi'njaam.

?i así es que ?iф ?eñč n-?êš-p ?a?y

and that is what $1 \mathrm{PRO}$ NEG $1 \mathrm{~A}: 3 \mathrm{O}-\mathrm{know}-\mathrm{FUT}$ if

ny-wi?pha:m

2A:3O-like

“"And so I don't know if you'll like that.",

(49) No dyim, eñch eeche wyat dyim,

no y-dim ?eñč ?e:če y-wat y-dim

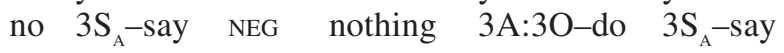

“"No," he says, “it doesn't matter.'

(50) Eñch eeche wyat.

?eñč ?e:če $y$-wat

NEG nothing 3A:3O-do

“ "It doesn't matter.'

(51) Ätz wää nwatta' yoos njem a'y chi' tokeñ.

?iф wi: n-wat-ta? yo:s nhem ?a?y či?

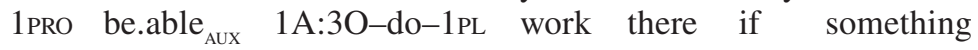

$\emptyset$-tokeñ

$3 \mathrm{~S}_{\mathrm{B}}$-be.lacking

"“We can do the work there if it is needed.' 
(52) Wää n'a'ayya'ta' dyim.

wi: $\quad$ n-?a?anya?-ta? $\quad \mathrm{y}$-dim

be.able AUx $_{1 \mathrm{~A}: 3 \mathrm{O}-\text { show-1PL } \quad 3 \mathrm{~S}_{\mathrm{A}}-\text { say }}$

" "We can show her.'

(53) Wää n'a'ayya'ta' njune'ep dyyoswat dyim.

wi: $\quad$ n-?a?anya?-ta? nhune?e-p y-yo?s-wat

be.able $_{\mathrm{AUX}}$ 1A:3O-show-1PL how-FUT $3 \mathrm{~S}_{\mathrm{A}}$-work-do

$$
\mathrm{y}-\operatorname{dim}
$$

$3 \mathrm{~S}_{\mathrm{A}}-\mathrm{say}$

" "We can show her how to work," he tells him.

(54) Usta'usta' byuska'ap dyim.

Pusta? ?usta? y-bus-ka?-p y-dim

DISTR little 3A:3O-learn-INTENS-FUT 3S $3 \mathrm{~S}_{\mathrm{A}}$-say

، "Little by little she's going to learn it," he says.'

(55) Byuska'ap dyim, njune'ep dyyoswat dyim.

y-bus-ka?-p y-dim nhune?e-p y-yo:s-wat

3A:3O-learn-INTENS-FUT $3 \mathrm{~S}_{\mathrm{A}}$-say how-FUT $3 \mathrm{~S}_{\mathrm{A}}$-work-do

$\mathrm{y}-\mathrm{dim}$

$3 \mathrm{~S}_{\mathrm{A}}-\mathrm{say}$

“"She's going to learn how to work," he says.'

(56) Bich njyaam dyim, a'y nshyun.

bic ny-ha:m y-dim ra?y ny-sun

2PRO 2A:3O-know 3S - say if 2A:3O-want

" "It's up to you," he says, "if you want her.",

(57) Pwes te nyakkä'nkomyaj dyim.

pues te n-yak-kînkom-yah y-dim

but INCL 1A:3O-CAUS-marry-3PL $3 \mathrm{~S}_{\mathrm{A}}$-say

“"Well, we're going to get them married," he says.'

(58) Pero ma'y knäma' dyim.

pero ma?-ya kn-dim-a? y-dim

but PERF-already 1A:2O-say-APPL $3 \mathrm{~S}_{\mathrm{A}}-$ say

، "But I told you," he says.'

(59) Yä'äpä' eñdye shyun yoswatp dyim.
yîipip
?eñdye $\mathrm{y}$-sun
$\emptyset$-yo:s-wat-p
$\mathrm{y}-\operatorname{dim}$
this
NEG
3A:3O-want
$3 \mathrm{~S}_{\mathrm{B}}$-work-do-FUT
$3 \mathrm{~S}_{\mathrm{A}}$-say

" "She doesn't want to work," he says.' 
(60) Si, pero yoom ma'y byatän ke njumbu' kañcheñ.

sí pero y-lo:m ma?-ya y-batin que

yes but 3PSR-father PERF-already 3A:3O-hear that

\section{nhumbu? $\emptyset$-kañčeñ}

very $\quad 3 \mathrm{~S}_{\mathrm{B}}-$ lazy

" "Yes, I heard from her father that she's very lazy.",

(61) Bweenu, ma' du'k tyempu jes kä'ykomp.

bueno ma? $\emptyset$-du?k tyempu hes $\emptyset$-ki?pkom-p

good PERF $3 \mathrm{~S}_{\mathrm{B}}$-arrive time when $3 \mathrm{~S}_{\mathrm{B}}$-marry-FUT

'Well, the time came for them to marry.'

(62) Ma' yakkä'ykomtabetz.

ma? $\emptyset$-yak-ki? kom-tah-be $\varnothing$

PERF $\quad 3 \mathrm{~S}_{\mathrm{B}}$-CAUS-marry-PASS-PL

'They got married.'

(63) Kä'ykomyaj.

$\emptyset$-ki?nkom-yah

$3 \mathrm{~S}_{\mathrm{B}}-$ marry-3PL

'They married.'

(64) Jmm, dyim, jeksh 'yaap ma' tzä'y njem.

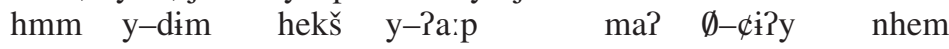

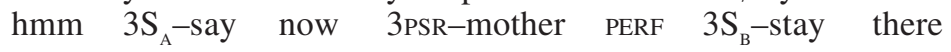

'Now the mother (of the bride) remained (having moved her daughter to the other house).'

(65) Ma' du'k chikä.

ma? $\emptyset-d u ' k \quad y-t i k-i$

PERF $3 \mathrm{~S}_{\mathrm{B}}$-arrive 3PSR-house-LOC

'(The couple) arrived at the house (of the groom).'

(66) Ma'te we'kyaje'.

ma?=te $\emptyset$-wê?k-yah-e?

PERF $=$ INC $3 \mathrm{~S}_{\mathrm{B}}-$ eat $-3 \mathrm{PL}-\mathrm{DEP}$

'They began to eat.'

(67) Byumbe ech.

y-bumbe $\emptyset$-?êč

3PSR-all $3 \mathrm{~S}_{\mathrm{B}}$-exist

'Everything was there.'

(68) Ma' säłkejyaj.

ma? $\emptyset$-sin-keh-yah

PERF $3 \mathrm{~S}_{\mathrm{B}}$-sun-appear-3PL

'The sun came up.' 
(69) Yoomä' u byon.

yo:mi? ?u y-boy

woman IMP $3 \mathrm{~S}_{\mathrm{A}}$-sleep

'The woman was sleeping.'

(70) Baytzun 'yaap.

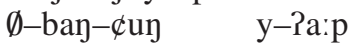

$3 \mathrm{~S}_{\mathrm{B}}$-stand-rise 3PSR-mother

'His mother woke up.'

(71) Ma'te dyyakwe'k shyuutu', dyim.

ma?=te $\quad y$-yak-wê?k $\quad y$-su:tu? $\quad y$-dim

PERF $=$ INC 3A:3O-CAUS-eat 3PSR-boy $3 \mathrm{~S}_{\mathrm{A}}$-say

'She began to feed her son.'

(72) Ma'y dyyakwe'k njem.

ma?-ya y-yak-wê?k nhem

PERF-already 3A:3O-CAUS-eat there

'There she fed him.'

(73) Ma' däk yooshuku.

ma? $\emptyset$-dik yo:s-yuku

PERF $3 \mathrm{~S}_{\mathrm{B}}-\mathrm{gO}$ work-LOC

'He went to work.'

(74) Kisa ya upna' dyyu'k dyim.

quizás ya ?upa-na? y-du?k y-dim

perhaps already now-still $3 \mathrm{~S}_{\mathrm{A}}$-arrive $3 \mathrm{~S}_{\mathrm{A}}$-say

“"Perhaps she's still coming (to learn)," (she) says.'

(75) Kisa byuska'ap yoos dyim.

quizás $y$-bus-ka?-p yo:s $y$-dim

perhaps 3A:3O-learn-INTENS-FUT work 3S $\mathrm{S}_{\mathrm{A}}$-say

“"Maybe she'll learn the work," (she) says.'

(76) Ma' bantzuy.

ma? $\emptyset$-ban- $\phi$ un

PERF $3 \mathrm{~S}_{\mathrm{B}}$-stand-rise

'She stood up.'

(77) Yoomä' ma' o'tzput.

yo:mi? ma? $\emptyset-$ - $0 ? \phi-$ put

woman PERF $3 \mathrm{~S}_{\mathrm{B}}$-sit-exit

'The woman went out to sit.'

(78) O'tzde'.

$\emptyset-$ - $0 ? \phi-$ de?

$3 \mathrm{~S}_{\mathrm{B}}$-sit-STV

'She sits.' 
(79) Ma'te peeyo'oye' chi'icho'om.

ma?=te $\emptyset-$ pe:y-?o?y-e? $\quad$-čê:ča?-o?o-m

PERF $=$ INC $3 \mathrm{~S}_{\mathrm{B}}$-rock-ANTIP-DEP 3PSR-hammock-LOC-LOC

'She begins to rock in the hammock.'

(80) Yä'äp yoomä' eñdye shyun yoswatp, dyim.

yîip yo:mi? ?eñdy y-sun ${ }^{y}$-yo:s-wat-p

this woman NEG 3A:3O-want $3 \mathrm{~S}_{\mathrm{B}}$-work-do-FuT

$$
\begin{aligned}
& \mathrm{y}-\mathrm{dim} \\
& 3 \mathrm{~S}_{\mathrm{A}}-\mathrm{say}
\end{aligned}
$$

“"This woman doesn't want to work," (her mother-in-law) says.'

(81) Ni sikyeera meñ kkä'pa't.

ni siquiera mêñ k-ki?-pa?t

NEG at.least come ${ }_{\mathrm{AUX}}$ 3A:10-hand-find

"'She doesn't even offer to help me.'

(82) Njune'ep meñ kkä'pa't? dyim.

nhune?e-p mêñ k-kî-pa?t $\quad \mathrm{y}$-dim

how-FUT come $_{\text {AUX }}$ 3A:1O-hand-find $3 \mathrm{~S}_{\mathrm{A}}$-say

"'How is she going to help me?" she says.'

(83) Eñdye shyun yoswatp.
?eñdye
$\mathrm{y}-$ sun
$\emptyset$-yo:s-wat-p
NEG
3A:3O-want
$3 \mathrm{~S}_{\mathrm{B}}$-work-do-FuT

“'She doesn't want to work.'

(84) Njune'ep nyakwe'kp, dyim,

nhune?e-p n-yak-wê?k-p y-dim

how-Fut 1A:30-CAus-eat-Fut 3S $3 \mathrm{~S}_{\mathrm{A}}$-say

“'How am I going to feed her," she asks,'

(85) ay meñche nyakwe'kp?

?åy mêñ-če n-yak-wê?k-p

if come $_{\mathrm{AUX}}$-LIM 1A:30-CAUS-eat-FUT

'“if she only comes for me to feed her?'

(86) Eñdye ma' bye'tz yoomä'? dyim.

Peñdye ma? y-be?ф yo:mi? y-dim

NEG PERF 3A:3O-look woman 3S -say

“"Hasn't he found his wife?" she asks.

(87) Sabe Dios!, nyakwe'k maan, dyim,

sabe dios n-yak-wê?k n-ba:n y-dim

knowat:3sG:PRES god 1A:3O-CAUS-eat 1PSR-child 3S $\mathrm{S}_{\mathrm{A}}$-say

“'God knows! I feed my son,” she says.' 
(88) poko biñ yooshuku, dyim,

porque y-bêñ yo:s-yuku y-dim

because $3 \mathrm{~S}_{\mathrm{A}}-$ come work-from $3 \mathrm{~S}_{\mathrm{A}}$-say

“"because my son is coming from work," she says,'

(89) I maan wää nyakwe'k, dyim,

?i n-ba:n wi: n-yak-wê?k y-dim

and 1PSR-child be.able ${ }_{\mathrm{AUX}}$ 1A:3O-CAUS-eat $3 \mathrm{~S}_{\mathrm{A}}-$ say

“"and I can feed my son," she says,'

(90) pero yä'äp yoomä', a'y eñdye shyun yoswatp.

pero yi̊ip yo:mi? ?a?y ?eñdye y-sun

but this woman if NEG 3A:30-want

$\emptyset$-yo:s-wat-p

$3 \mathrm{~S}_{\mathrm{B}}$-work-do-FUT

" "but this woman doesn't even want to work.",

(91) Pwe te npuuta'ap eyäm dyim, maas seguuru dyim.

pues te $n-$ put-Da?-p Reyim $y-$ dim ma:s

well INCL 1A:3O-exit-CAUS-FUT apart 3S $\mathrm{A}_{\mathrm{A}}$-say more

$$
\text { seguro } \mathrm{y}-\mathrm{dim}
$$

secure $3 \mathrm{~S}_{\mathrm{A}}-$ say

“"Well, we're going to separate her, it's a better bet," (the boy's father) says."

(92) Joye nwästäkä te npuuta'ap dyim.

hoye nwis-tik-i te n-put-Da?-p y-dim

morning two-NCLF-LOC INCL 1A:3O-exit-CAUS-FUT 3S $\mathrm{A}_{\mathrm{A}}$-say

“"Tomorrow at two, I'm going to take her out," (the boy's mother) says.'

(93) Che'ejä' pilaat dyim, byumbe kya'ache' dyiiksa'aje'.

čê?-h-i? y-pila:t y-dim y-bumbe y-ka?ače?

give-LIG-IMP 3PSR-plate 3S $\mathrm{S}_{\mathrm{A}}$-say 3PSR-all 3PSR-utensils

$$
\text { y-diks-Da?-h-e? }
$$

3A:3O-go-CAUS-LIG-DEP

“"Give her her dishes!” she says (to her husband), "she can take all her stuff.",

(94) I ma'wye'kput tuykak täk.

?i ma? y-we?k-put tuykak tik and PERF 3A:3O-separate-exit other house

'And she moved her to another house.' 
(95) Wyata' chik.

y-wat-a? $\quad$-tik

3A:3O-do-APPL 3PSR-house

'She made her house.'

(96) Ma' dyiiksa' kya'ache', byumbe kya' ache'.

ma? y-diks-Da? y-ka?ače? y-bumbe y-ka?ače?

PERF 3A:3O-go-CAUS 3PSR-utensils 3PSR-all 3PSR-utensils

'(The mother-in-law) took her kitchen stuff, all her stuff.'

(97) Shyu'un, byajko', byumbe, kyasweela', pilaat, boy tzakätaje' njem.

y-su?un y-bah-ko? y-bumbe y-casuela y-pila:t

3PSR-pot 3S - give-INSTR 3PSR-all 3PSR-pan 3PSR-plate

$\begin{array}{lll}\text { boy } & \emptyset-\phi a k-a 2-h-e ? & \text { nhem } \\ \text { go.and.return }_{\text {AUX }}: \text { PERF } & 3 \mathrm{~S}_{\mathrm{B}}-\mathrm{leave}-\mathrm{APPL}-\mathrm{LIG}-\mathrm{DEP} & \text { there }\end{array}$

'Her pot, she gave it all, her pan, dishes, she went to leave them there.'

(98) Ta'tztzakätaj bäkske pälaat.

$\begin{array}{lll}\emptyset-\text { ta? } \phi-\not a k-a ?-t a h & \text { biks-ke } & \text { pila:t } \\ 3 \mathrm{~S}_{\mathrm{B}} \text {-stow-leave-APPL-PASS } & \text { much-INTENS } & \text { plate }\end{array}$

'Left many dishes stowed (there).'

(99) Maan dyim, njem ech dyim,

n-ba:n y-dim nhem $\emptyset$-rêč $\quad \mathrm{y}$-dim

1PSR-child $3 \mathrm{~S}_{\mathrm{A}}$-say there $3 \mathrm{~S}_{\mathrm{B}}$-be $3 \mathrm{~S}_{\mathrm{A}}$-say

“"My son," she says, "there it is,'

(100) basta nkya'ache'.

basta ny-ka?ače?

much 2PSR-utensils

“'many (kitchen) utensils.'

(101) Basta n'iicha' nkya'ache'.

basta ny-?êč-Da? ny-ka?ače?

much 2A:3O-be-CAUS 2PSR-utensils

" "You have many utensils.",

(102) Si dyim, wää ich.

sí $\quad \mathrm{y}-\mathrm{dim}$ wi: $\quad \mathrm{y}-$ ?êč

yes $3 \mathrm{~S}_{\mathrm{A}}$-say good $3 \mathrm{~S}_{\mathrm{A}}$-is

" "Yes," he says, "it's fine.",

(103) Jeksh jepe' pääñ ma'te put eyäm.

hekš hepe? pi:ñ ma?=te $\emptyset$-put ?eyim

now this man PERF=INC $3 \mathrm{~S}_{\mathrm{B}}$-leave apart

'Now this man leaves.' 
(104) Ma'y shyos byo'os byumbe.

$\begin{array}{llll}\text { ma?=ya } & \text { y-sos } & \text { y-boios } & \text { y-bumbe } \\ \text { PERF=already } & \text { 3A:3O-cook } & \text { 3PSR-nixtamal } & \text { 3PSR-all }\end{array}$

'(The woman) finished preparing her nixtamal, all of it.'

(105) Ma'te waaye'. Ma' dyyaj wyay.

ma?=te $\emptyset$-way-e? ma? y-yah y-way

PERF=INC $\quad 3 \mathrm{~S}_{\mathrm{B}}-$ grind-DEP PERF $3 \mathrm{~A}: 3 \mathrm{O}-$ finish $\quad 3 \mathrm{~S}_{\mathrm{A}}$-grind

'She began grinding. She finished grinding.'

(106) Pwes ma' meñ dyya'an yooshuku, dyim,

pues ma? $\emptyset$-mêñ y-da?ay yo:s-juku y-dim

well PERF $3 \mathrm{~S}_{\mathrm{B}}$-come 3PSR-husband work-from $3 \mathrm{~S}_{\mathrm{A}}-$ say

'Well, her husband came from work, he says,'

(107) Upa nwe'k dyim.

?upa n-wê?k $\mathrm{y}$-dim

now $1 \mathrm{~S}_{\mathrm{A}}$-eat $3 \mathrm{~S}_{\mathrm{A}}-$ say

“ "Now I'm going to eat," he says.'

(108) Byeja, upa nwe'k!

vieja Pupa n-wê?k

old.woman now $1 \mathrm{~S}_{\mathrm{A}}$-eat

،"Wife, now I'm going to eat!",

(109) Upa nwi'k? dyim.

Pupa ny-wê?k y-dim

now $2 \mathrm{~S}_{\mathrm{A}}$-eat $3 \mathrm{~S}_{\mathrm{A}}$-say

“"Now you're going to eat?" she asks.'

(110) Upa nwe'k dyim.

Pupa n-wê?k $\mathrm{y}$-dim

now $1 \mathrm{~S}_{\mathrm{A}}$-eat $3 \mathrm{~S}_{\mathrm{A}}-$ say

“"Now I'm going to eat.",

(111) Ma'te dyyakwe'k.

ma?=te $\quad \mathrm{y}-\mathrm{ak}-\mathrm{wê}\} \mathrm{k}$

PERF $=$ INC 3A:3O-CAUS-eat

'She began to feed him.'

(112) Ma'ye wi'k jepe' pääñ, byumbe.

ma?-ye $\quad \mathrm{y}$-wê?k hepe? pi:ñ y-bumbe

PERF-already $3 \mathrm{~S}_{\mathrm{A}}$-eat this man 3PSR-all

'The man finished eating, everything.' 
(113) I yoomä'. Ma'y wi'kyaj wistäk.

?i yo:mi? ma?-ya y-wê?k-yah y-wis-tik

and woman PERF-already $3 \mathrm{~S}_{\mathrm{A}}-$ eat-3PL $3 \mathrm{~S}_{\mathrm{A}}-\mathrm{tWO}-\mathrm{NCLF}$

'And (also) the woman. The two of them ate.'

(114) Dyim, Jeksh dyim, yä'äp pälaat, ee chye'eka'aj? dyim.

y-dim hekš $y$-dim yịip pila:t

$3 \mathrm{~S}_{\mathrm{A}}$-say now $3 \mathrm{~S}_{\mathrm{A}}-$ say this plate

Pe: $\quad \mathrm{y}-\phi \mathrm{e}$-ka?ah $\quad \mathrm{y}-\mathrm{dim}$

who 3A:3O-wash-INTENS:Fut $3 \mathrm{~S}_{\mathrm{A}}$-say

"He says, "Now, these dishes, who's going to wash them?" he asks.'

(115) Njem däk tzaakä' komjoso'om dyim.

nhem dik $\phi$ ak-i? kom-yos-oro-m y-dim

there $\mathrm{go}_{\mathrm{AUX}}$ leave-IMP post-hole-LOC-LOC $3 \mathrm{~S}_{\mathrm{A}}$-say

“'Leave it there in the corner!" he says.'

(116) Jembesh te npu'utja'ap kwyenda dyim.

hembe?-eš te n-put-Da?-h-a?-p y-cuenta

there-FUT INCL 1A:3O-exit-CAUS-LIG-APPL-FUT 3PSR-count

$\mathrm{y}-\mathrm{dim}$

$3 \mathrm{~S}_{\mathrm{A}}-$ say

“"There we're going to take count," he says.'

(117) Te maychu'una'ap nju'utza ech dyim.

te n-bay-tun-?a?-p nhu?ựa $\emptyset$-?êč

INCL 1A:3O-count-road-VBZR-FUT how.many $3 \mathrm{~S}_{\mathrm{B}}$-be

$\mathrm{y}-\mathrm{dim}$

$3 \mathrm{~S}_{\mathrm{A}}-$ say

“"We're going to count how many there are.",

(118) Njemesh ta mayka'ps dyim.

nhem-eš ta n-bay-ka?ps y-dim

there-FUT INCL 1A:3O-count-accomplish $3 \mathrm{~S}_{\mathrm{A}}$-say

“"There we're going to count," he says.'

(119) Tzaakä'!

$\emptyset-c \mathrm{ak}-\mathrm{i} ?$

3PO-leave-IMP

" "Leave it!",

(120) Bueeno.

'Good.' 
(121) Chyak yoomä'.

y-cak yo:mi?

3A:3O-leave woman

'The woman left them.'

(122) Tsu'yn'eechkak eep ma'te we'eke'.

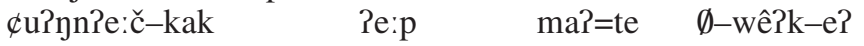

in.the.afternoon-REPET other.time $P E R F=I N C \quad 3 S_{B}-$ eat-DEP

'In the afternoon, again he began to eat.'

(123) Ma' du'k, ma'te we'kyaje'.

ma? $\emptyset$-durk ma?=te $\emptyset$-wê?k-yah-e?

PERF $3 \mathrm{~S}_{\mathrm{B}}$-arrive $\quad$ PERF $=\mathrm{INC} \quad 3 \mathrm{~S}_{\mathrm{B}}$-eat $-3 \mathrm{PL}-\mathrm{DEP}$

'He arrived (and) began to eat.'

(124) Ma' we'kyaj wistäk.

ma? $\emptyset$-wê?k-yah y-wis-tik

PERF $3 \mathrm{~S}_{\mathrm{B}}-$ eat-3PL $3 \mathrm{~S}_{\mathrm{A}}-\mathrm{twO}-\mathrm{NUMC}$

'The two of them ate.'

(125) Eepä' dyimäkak dyim,

Re:pi? y-dim-a?-kak y-dim

other.time 3A:3O-say-APPL-REPET $3 \mathrm{~S}_{\mathrm{A}}$-say

'Again when he comes back he says,'

(126) Bweenu yä'äp pälaat, ee chye'eka'aj? dyim.

bueno yirip pila:t ?e: y-ce?-ka?ah y-dim

good these plate who 3A:30-wash-INTENS:FUT $3 \mathrm{~S}_{\mathrm{A}}$-say

“"Okay, who's going to wash these dishes?" he says.'

(127) Njem däk tzaakä' dyim, komjoso'om! dyim.

nhem dik cak-i? y-dim kom-hos-o?o-m

there toward.there leave-IMP $3 \mathrm{~S}_{\mathrm{A}}$-say post-hole-LOC-LOC

$$
\mathrm{y}-\operatorname{dim}
$$

$3 \mathrm{~S}_{\mathrm{A}}-$ say

“"Leave them there in the corner!" he says.'

(128) Njemesh ta mayka'ps dyim.

nhem-ě̌ ta n-bay-ka?ps

there-FUT INCL 1A:3O-count-accomplish 3S $\mathrm{S}_{\mathrm{A}}$-say

" "There we're going to count them.",

(129) Bweenu, ma' chya'tztzak.

bueno ma? $y-t a ? \phi-c a k$

good PERF 3A:3O-load-leave

“"Good, we'll leave them piled up.", 
(130) Ma' kej eech.

ma? $\emptyset$-keh ?ěč-D

PERF $3 \mathrm{~S}_{\mathrm{B}}$-appear be-NOM

'He woke up.'

(131) Jembe' shamaan ma' dyyaj.

hembe? šama:n ma? y-yah

there week PERF 3A:3O-end

'There the week ended.'

(132) Ma' dyyajyaj pooy.

ma? y-yah-yah po:y

PERF 3A:3O-finish-3PL mes

'Months went by.'

(133) Kyak wiñ pääñ.

y-kak y-wêñ pi:ñ

3A:3O-change $3 \mathrm{~S}_{\mathrm{A}}-\mathrm{REFL}$ man

'The man was changing.'

(134) I ñyo't, ee kchye'eka'ya'ap?

?i ny-yo?t ?e: ky- фe?-ka?y-a?-p

and 2PSR-clothes who 3A:2O -wash-INTENS-APPL-FUT

“"And your clothes, who's going to wash them for you?",

(135) Njem tzaakä' nee nje'!

nhem cak-i? ne: nhe?

there leave-IMP also 3PRO

"'Leave that there too!'

(136) Njemesh ta mayka'ps dyim.

njem-eš ta n-bay-ka?ps y-dim

there-FUT INCL 1A:3O-count-accomplish $3 \mathrm{~S}_{\mathrm{A}}$-say

“"There we're going to count," he says.'

(137) Bweenu, pyatztzaka' dyyo't, dyyojkoy.

bueno $\mathrm{y}$-pa $\not<-\not \mathrm{ak}=\mathrm{am} \quad \mathrm{y}$-yo?t $\quad \mathrm{y}$-dohkoy

good 3A:3O-throw-leave=IAM 3PSR-clothes 3PSR-pants

'Well, he left his shirt, his pants.'

(138) Kyak wiñ nee yoomä'.

$\mathrm{y}-\mathrm{kak}$

y-wêñ

ne: yo:mi?

3A:3O-change $3 \mathrm{~S}_{\mathrm{A}}-\mathrm{REFL}$ also woman

'The woman changed too.' 
(139) I nyo't dyim, ee ktze'eka'ya'ap?

?i n-yo?t y-dim Pee k-ce?-ka?y-a?-p

and 1PSR-clothes 3S-say who 3A:1O-wash-INTENS-APPL-FUT

“"And my clothes?" (the girl) asks, "Who's going to wash them for me?",

(140) Njem tzaakä'!

nhem фak-i?

there leave-IMP

" "Leave them there!'

(141) Njemesh ta mayka'ps dyim.

nhem-eř ta n-bay-kaips y-dim

there-FUT INCL 1A:3O-count-accomplish $3 \mathrm{~S}_{\mathrm{A}}$-say

“"There we'll take a count," he says.'

(142) Aa, wää dyim.

a: wi: $\quad y-\operatorname{dim}$

ah good $3 \mathrm{~S}_{\mathrm{A}}$-say

“"Ahh, that's fine," she says.'

(143) Ma' chyak.

ma? $y-\not a k$

PERF 3A:3O-leave

'She left it.'

(144) Byumbe upche chyuup. Upche chyuup kya'ache'.

y-bumbe Pupa-če y-tu:p ?upa-če y-tu:p

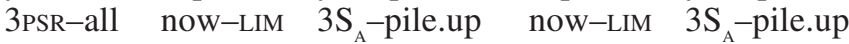

y-ka?ače?

3PSR-utensils

'Everything was piling up. The dishes were piling up.'

(145) Upche chyuup.

?upa-če y-tu:p

now-LIM 3A:3O-pile.up

'Now it was piled up.'

(146) Jeksh pääñ dyyos'a'. Dyim,

hekš pi:ñ y-yos-?a?m y-dim

now man 3A:3O-be.obliged-look 3S - say

'Now the man looks. He says,'

(147) Paa su gaayu, yä'äp eñdye shyun tze'ejo'oj!

para su gallo yi̊ip ?eñ $\mathrm{d}^{\mathrm{y}} \mathrm{e}$ y-sun

for his rooster this NEG 3A:3O-want

$\emptyset-c \mathrm{e}-\mathrm{h}-\mathrm{Po}$ oh

$3 \mathrm{~S}_{\mathrm{B}}$-Wash-LIG-ANTIP:FUT

“"Gosh, this one doesn't want to clean!' 
(148) Eñdyee shyun chye'ep pälaat! dyim.

$\begin{array}{lllll}\text { Peñdye } & y-\text { sun } & y-\phi e ?-p & \text { pila:t } & \text { y-dim } \\ \text { NEG } & 3 \mathrm{~A}: 3 \mathrm{O}-\text { want } & 3 \mathrm{~A}: 3 \mathrm{O}-\text { wash-Fut } & \text { plate } & 3 \mathrm{~S}-\text { say }\end{array}$

" "She doesn't want to wash the dishes," he says (to himself).'

(149) I nojkoy, ya ma' yaj nojkoy.

?i n-dohkoy ya ma? Ø-yah n-dohkoy and 1PSR-pants already PERF $3 \mathrm{~S}_{\mathrm{B}}$-finish 1PSR-pants

“"And my pants! I have no more pants!'

(150) Ma' yajam nka'ache'.

ma? $\emptyset-$ yah=am n-ka?ače?

PERF $3 \mathrm{~S}_{\mathrm{B}}$-finish=IAM 1PSR-utensils

“"There are no more utensils.'

(151) Njem tuupde' ij!

nhem $\emptyset$-tu:p-de? y-?êč

there $\quad 3 \mathrm{~S}_{\mathrm{B}}$-pile.up-STV $3 \mathrm{~S}_{\mathrm{A}}-$ be

" "There they are piled up!",

(152) Dyim, wää ich dyim,

y-dim wi: $\quad y-$-?êč $\quad y-d i m$

$3 \mathrm{~S}_{\mathrm{A}}$-say good $3 \mathrm{~S}_{\mathrm{A}}$-be $3 \mathrm{~S}_{\mathrm{A}}$-say

'He says, "That's fine," he says,'

(153) Joye kbej. Joye ish che' nwata'ap dyim.

hoye k-beh hoye y-?êš

tomorrow $1 \mathrm{~S}_{\mathrm{B}}$-come:FuT tomorrow 3A:30-see

če? n-wat-a?-p y-dim

that $1 \mathrm{~A}: 3 \mathrm{O}-\mathrm{do}-\mathrm{APPL}-\mathrm{FUT} \quad 3 \mathrm{~S}_{\mathrm{A}}-\mathrm{say}$

“ "Tomorrow I'll come. Tomorrow she'll see what I'm going to do," he says.'

(154) Tzaakä'!

cak-i?

leave-IMP

“"Leave it!",

(155) Ma' däk yoswaache'.

ma? dik $\emptyset$-yo:s-wat-e?

PERF $\quad$ go $_{\text {AUX }} \quad 3 \mathrm{~S}_{\mathrm{B}}$-work-do-DEP

'He went to work.'

(156) Ma'jiikput chyujku', ma' däkam.

ma? y-hi:k-put ma?

PERF 3A:3O-pull-exit 3PSR-shoot-INSTR.NOM PERF

$\emptyset$-dik=am

$3 \mathrm{~S}_{\mathrm{B}}-\mathrm{gO}=\mathrm{IAM}$

'He pulled out his gun out and went.' 
(157) Ku'kp jaa biñ.

ku?k-p ha: y-bêñ

middle-ADJZ day $3 \mathrm{~S}_{\mathrm{A}}-$ come

'At midday he came.'

(158) Upa nwe'k, byeja.

?upa n-wê?k vieja

now $1 \mathrm{~S}_{\mathrm{A}}$-eat old.woman

، "Now I'm going to eat, wife.",

(159) Upa nwi'k?

?upa ny-wê?k

now $2 \mathrm{~S}_{\mathrm{A}}$-eat

“"Now you're going to eat?",

(160) Upa nwe'k.

?upa n-wê?k

now $1 \mathrm{~S}_{\mathrm{A}}$-eat

، “Now I'm going to eat.'

(161) Dejde yä'äp jaa eñdye nsun ekä k'oota'ap.

desde yîip haa ?eñdye n-sun ?eki k-?o:ta?-p

since this day NEG 1/3-want that 1A:3O-speak-FUT

“"From now on, I don't want (anyone) to speak to me.'

(162) Ootäpaapä', dyim, ntujka'ap.

$\emptyset-$ - o:ta?-pa:=pi? $\quad \mathrm{y}-\mathrm{dim} \quad \mathrm{n}-\mathrm{tuh}-\mathrm{ka}-\mathrm{p}$

$3 \mathrm{~S}_{\mathrm{B}}$-speak-NOM=REL $3 \mathrm{~S}_{\mathrm{A}}$-say $1 \mathrm{~A}: 3 \mathrm{O}-$-shoot-INTENS-FUT

" "He who speaks," he says, "I will shoot them (or him/her/it).",

(163) Pyatztzak chyujku'.

y-paф-cak y-tuh-ku?

3A:3O-shoot-leave 3PSR-shoot-INSTR.NOM

'He left his gun (lying in front of him).'

(164) Ootäpaapä' ntujka'ap.

$\emptyset-$-?o:ta?-pa:=pi? n-tuh-ka?-p

$3 \mathrm{~S}_{\mathrm{B}}$-speak-NOM=REL 1A:3O-shoot-INTENS-FUT

،"He who speaks, I will shoot.'

(165) K'aạwejäpaapä' nee nje' ntujka'ap.

k-?a:yweh-a?-pa:=pi? ne: nhe?

3A:1O-shout - APPL - NOM=REL also 3PRO

n-tuh-ka?-p

1A:3O-shoot-INTENS-FUT

“"He who shouts at me, I will also shoot.' 
(166) Eñchpä' yoswatp, nee nje' ntujka'ap.

$$
\begin{array}{llll}
\text { Reñč=pi? } & \emptyset \text {-yo:s-wat-p } & \text { ne: } & \text { nhe? } \\
\text { NEG=REL } & 3 S_{\text {B }} \text {-work-do-FUT } & \text { also } & 3 \text { PRO } \\
\text { n-tuh-ka-p } & & \\
\text { 1A:3O-shoot-INTENS-FUT } & &
\end{array}
$$

“"He who doesn't work, I will also shoot.'

(167) K'wanäpaapä' nee nje' ntujka'ap.

k-wan-a?-pa:=pi? ne: nhe? n-tuh-ka?-p

3A:1O-sing-APPL-NOM=REL also 3PRO 1A:3O-shoot-INT-FUT

“"Whoever sings to me, I will also shoot.'

(168) Eñche nsun ee meñ k'oota'.
?eñče n-sun ?e:
mêñ k-3o:ta?
NEG 1A:3O-want someone come AUX $_{\text {3 }}$ 3A:1O-speak

'“I don't want anyone to come speak to me.'

(169) Eñdyee nsun ni k'oota'ap, ni k'aanweja'ap nee eena',

Peñdye: n-sun ni k-?o:ta?-p

NEG 1A:3O-want NEG 3A:1O-speak-FUT

$$
\begin{array}{llll}
\text { ni } & \text { k-Pa:yweh-ai-p } & \text { ne: } & \text { Pe:-na } \\
\text { NEG } & \text { 3A:1O-shout-APPL-FUT } & \text { NEG } & \text { someone-still }
\end{array}
$$

'"Nor do I want anyone to speak to me, nor shout at me,'

(170) poko ntujka'ap.

poco n-tuy-ka?-p

because 1A:3O-shoot-INTENS-FUT

" "because I will shoot them.",

(171) Juuta! Jep ma' byatäy jepe' yoomä'.

hu:ta hep ma? h-batin hepe? yo:mi?

EXCL this PERF 3A:3O-hear this woman

'Oh my goodness! The woman heard this.'

(172) Poytzuy njem.

$\emptyset$-poy- $\not$ un nhem

$3 \mathrm{~S}_{\mathrm{B}}$-run-rise there

'There she ran.'

(173) Pyuuta' pilaat.

y-put-Da? y-pila:t

3A:3O-exit-CAUS 3PSR-dishes

'She took out her dishes.'

(174) Ma'te chye'tze'ka'y.

ma? =te $\quad \mathrm{y}-\phi \mathrm{e} ? \sim \not \mathrm{e}$ ?-ka?y

PERF $=$ INC 3A:3O-DISTR $\sim$ Wash-INTENS

'She began to wash.' 
(175) A'y eñch k'yoswatp dyim, ktujka'ap yä'äp. Pa?y Reñč k-yo:s-wat-p y-dim k-tuh-ka?-p if $\quad \mathrm{NEG} \quad 1 \mathrm{~S}_{\mathrm{B}}$-work-do-FUT $3 \mathrm{~S}_{\mathrm{A}}$-say $3 \mathrm{~A}: 1 \mathrm{O}-$-shoot-INT-FUT yiłip this

" "If I don't work," she says, "this one is going to shoot me.",

(176) K'yaka'ap' dyim.

k-yaka?-p $\quad \mathrm{y}$-dim

3A:1O-kill-FuT $3 \mathrm{~S}_{\mathrm{A}}$-say

"'He is going to kill me," she says.'

(177) Jekshejekshe chye'tze'ka'y chye'tze'ka'y.

hekšehekše $y-\not e ? \sim \not e ?-k a ? y$

rapid 3A:3O-DISTR Wash-INTENS

$\mathrm{y}-\not \mathrm{e} ? \sim \phi \mathrm{e}-\mathrm{ka} \mathrm{y}$

3A:3O-DISTR Wash-INTENS

'Quickly, she washed (and) she washed.'

(178) Ma'y wi'k pääñ.

ma?=ya $\quad$-wê?k pi:ñ

PERF=already $3 \mathrm{~S}_{\mathrm{A}}$-eat man

'The man finished eating.'

(179) Putam.

$\emptyset$-put=am

$3 \mathrm{~S}_{\mathrm{B}}$-exit=IAM

'He left.'

(180) Jeksh upche dyyos'a', dyim.

hekš ?upa-če y-yos-?a?m y-dim

now now-Lim 3A:3O-be.obliged-look 3S -say

'Now he's looking at her, he says,'

(181) Ya be jepe' shyun dyim.

ya ve hepe? y-sun $\quad \mathrm{y}$-dim

already see:IMP this 3A:30-want $3 \mathrm{~S}_{\mathrm{A}}$-say

"'You see, this is what he wants," he says.'

(182) Entonse eñch wää ta naks yoomä' dyim.

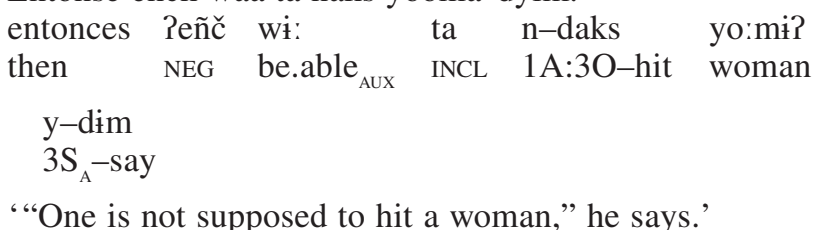


(183) Njune'ep naks? dyim.

nhune?e-p n-daks $\quad y$-dim

how-Fut 1A:3O-hit $3 \mathrm{~S}_{\mathrm{A}}$-say

" How could I hit her?",

(184) Jwasilitu dyim, pa ta nwatp dyim.

facilito $y-d i m$ pa ta $n-w a t-p \quad y-d i m$

easy $3 \mathrm{~S}_{\mathrm{A}}$-say to INCL $1 \mathrm{~A}: 3 \mathrm{O}-$ do-FUT $3 \mathrm{~S}_{\mathrm{A}}$-say

“"It's easy to do," he says.'

(185) Ta numwatp dyim.

ta n-duumu?-wat-p $\quad y-d i m$

INCL 1A:3O-straight-do-Fut $3 \mathrm{~S}_{\mathrm{A}}$-say

“"We're going to straighten her out," he says.'

(186) Jwasilitu ta numwatp tum pääñ dyim.

facilito ta n-du:mu?-wat-p tum pi:ñ y-dim

easy INCL 1A:3O-straight-do-FUT one man $3 \mathrm{~S}_{\mathrm{A}}$-say

“"It's easy to straighten out a man," he says.'

(187) Tum yoomä', tum pääñ, byumbe ta numwatp dyim.

tum yo:mi? tum pi:?ñ y-bumbe ta

one woman one man 3PSR-all INCL

$$
\text { n-du:mu?-wat-p } \quad \mathrm{y}-\mathrm{dim}
$$

1A:3O-straight-do-FuT $3 \mathrm{~S}_{\mathrm{A}}$-say

“"A woman, a man, everyone can be straightened out," he says.'

(188) Wää ij.

wi: $\quad \mathrm{y}-$ ?êč

good $3 \mathrm{~S}_{\mathrm{A}}-\mathrm{be}$

'That's fine.'

(189) Limpiu ma' tzä'y kya'ache'.

limpio ma? $\emptyset$-çîy $\quad$-ka?ače?

clean PERF $3 \mathrm{~S}_{\mathrm{B}}$-stay 3PSR-utensils

'The kitchen utensils ended up clean.'

(190) Pyetpyetka'y chik.

y-pet pet-ka?y y-tik

3A:3O-DISTR Sweep-INTENS 3PSR-house

'She swept the house there.'

(191) Njem byumbe chyak.

nhem $\mathrm{y}$-bumbe $\mathrm{y}$ - $\mathrm{c}$ ak

there 3PSR-all 3A:3O-leave

'She left it all there.' 
(192) Ma' täkeñ jojo.

ma? $\emptyset$-tikeñ hoho

PERF $3 \mathrm{~S}_{\mathrm{B}}-$ enter inside

'He entered.'

(193) Upa nwe'kp.

?upa n-wê?k-p

now $1 \mathrm{~S}_{\mathrm{A}}$-eat-FuT

، "Now I'm going to eat.'

(194) Aa, wää.

a: wi:

ah good

، “Ah, that's good.'

(195) Ma'te tze'jo'oye'.

ma?=te $\emptyset-c e ?-h-$ - o? - -?

PERF $=I N C \quad 3 \mathrm{~S}_{\mathrm{B}}-$ clean-LIG-ANTIP-DEP

“ "Now she's going to clean.'

(196) Chye'ejo'y.

$\mathrm{y}-\mathrm{ce}$ ?-h-?o?y

$3 \mathrm{~S}_{\mathrm{A}}$-clean-LIG-ANTIP

" "She's cleaning.",

(197) Ma' chyak chye'eko' njem, ma' däk boy dyyakwe'k dyya'an.

ma? $y-c a k \quad y-c e ?-k o ? \quad$ nhem

PERF 3A:3O-leave 3PSR-wash-INSTR there

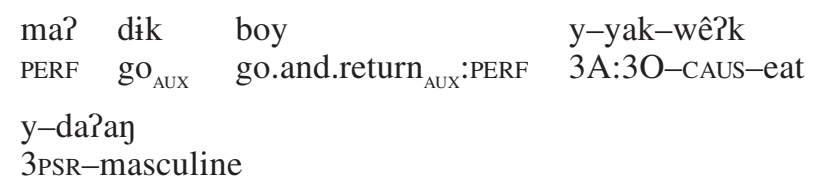

'(Later) she left her washing (and) went to feed her husband.'

(198) Ma'ye wi'k njem.

ma?=ya $\quad \mathrm{y}$-wê?k nhem

PERF=already $3 \mathrm{~S}_{\mathrm{A}}$-eat there

'There he began to eat.'

(199) Put ma' däk weeche'.

$\emptyset$-put ma? dik $\emptyset$-wêč-e?

$3 \mathrm{~S}_{\mathrm{B}}$-leave PERF $\mathrm{go}_{\mathrm{AUX}} \quad 3 \mathrm{~S}_{\mathrm{B}}$-walk-DEP

'He left to go for a walk.' 
(200) Aoora si dyim, nuuñe' upam dyyoswat dyim.

ahora sí $y$-dim n-du:ñe? ?upa=am y-yo:s-wat now yes $3 \mathrm{~S}_{\mathrm{A}}$-say $1 \mathrm{PSR}-$ woman now=IAM $3 \mathrm{~S}_{\mathrm{A}}$-work-do $\mathrm{y}-\operatorname{dim}$ $3 \mathrm{~S}_{\mathrm{A}}-\mathrm{say}$

“"Now, yes," he says, "my wife works," he says.'

(201) Aa, kinse diia ma' dyyaj.

a: quince día ma? y-yah

aa fifteen day PERF 3A:3O-finish

'Fifteen days passed.'

(202) Ma' du'k 'yoom yomsuutu'.

ma? $\emptyset$-du?k y-?o:m yo:mi?•su:tu?

PERF $3 \mathrm{~S}_{\mathrm{B}}$-arrive 3PSR-father woman•young

'The father of the young woman arrived.'

(203) Chi' nwyat, mä'ät?

či? ny-wat n-birit

what 2A:3O-do 1PSR-son.in.law

"“What are you doing, son-in-law?",

(204) Che' däk nwat? dyim.

če? dik n-wat y-dim

what $\mathrm{go}_{\mathrm{Aux}}$ 1A:30-do $3 \mathrm{~S}_{\mathrm{A}}$-say

“"What am I going to do?" he says.'

(205) Yä'ä k'ech npaakdas dyim.

yîi k-?êč n-pa:k-das y-dim

here $1 \mathrm{~S}_{\mathrm{B}}$-be $1 \mathrm{~S}_{\mathrm{A}}$-cold-PASs $3 \mathrm{~S}_{\mathrm{A}}$-say

“"Here I am refreshing myself," he says.'

(206) Aa, ma' kyakpak wiñ pääñ.

a: ma? y-kak-pak $\quad$ y-wêñ pi:ñ

aa PERF 3A:3O-change-have 3PSR-SELF man

'Ah, the man is well-dressed.'

(207) Dyaanche yä'äp mä'ät dyim, kyakpak wiñ.

$\mathrm{d}^{\mathrm{y}}$ a:nče yi?ip n-bi?it $\quad \mathrm{y}$-dim

wow this 1PSR-son.in.law $3 \mathrm{~S}_{\mathrm{A}}-$ say

y-kak-pak $\quad \mathrm{y}$-wêñ

3A:3O-change-have 3PSR-SELF

“"Wow, this son-in-law of mine," he says, "he's dressed himself well.", 
(208) Ätz asta njunu n'ech dyim.

?ił hasta nhunu n-?êč $y-d i m$

1 PRO until how $1 \mathrm{~S}_{\mathrm{A}}-$ be $3 \mathrm{~S}_{\mathrm{A}}-$ say

“"Me, now (look) how I am," he says.'

(209) Asta ke eñdya k'wää dyim.

hasta que ?eñdya k-wi: $\quad \mathrm{y}$-dim

until that NEG $1 \mathrm{~S}_{\mathrm{B}}-$ good $3 \mathrm{~S}_{\mathrm{A}}$-say

“"I'm not (doing) well," he says.'

(210) Dyim, mä'ät, ma'y kyak wiñ dyim.

y-dim n-birit ma?-ya y-kak

$3 \mathrm{~S}_{\mathrm{A}}$-say 1PSR-son.in.law PERF-already 3A:3O-change

y-wêñ $\quad y-d i m$

3PSR-REFL 3S - say

'He says, "My son-in-law has changed himself," he says.'

(211) N'a'mka' chiko'o.

n-?a?m-ka?m y-tik-o?o

1A:3O-look-enter 3PSR-house-LOC

“'I looked in his house.'

(212) Ya ma'ye pyetpyetjo'y nyombaa.

ya ma?=ye $y$-pet $\sim$ pet-ho?y n-yo:mi?-ba:

already $\quad$ PERF=already $3 \mathrm{~S}_{\mathrm{A}}$-DISTR $\sim$ Sweep-AND $\quad 1 \mathrm{PSR}-$ woman-DIM

“'My daughter has gone about sweeping.'

(213) Njune' ma' wyat, dyim, yä'äjä'?

nhune? ma? y-wat $y$-dim yirihi?

how PERF 3A:3O-do 3S -say this

" "How did he do this?" he says (to himself).'

(214) Bweenu, bä'ät dyim, njune' ma' nwyat dyim, ekä nyombaa ke yoswatp? dyim.

bueno birit $y$-dim nhune? ma? ny-wat

good son.in.law $3 \mathrm{~S}_{\mathrm{A}}$-say how PERF 2A:3O-do

y-dim Reki n-yo:mir-ba: que $\emptyset$-yo:s-wat-p

$3 \mathrm{~S}_{\mathrm{A}}$-say that 1PSR-woman-DIM that $3 \mathrm{~S}_{\mathrm{B}}$-work-do-FUT

y-dim

$3 \mathrm{~S}_{\mathrm{A}}$-say

“"Well, son-in-law," he says, "how did you do this, that my daughter is a worker?" he says.'

(215) Pero njune'ep maas dyim.

pero nhune?e-p mas $y$-dim

but how-Fut more $3 \mathrm{~S}_{\mathrm{A}}$-say

“"But how?" he asks.' 
(216) Kreo ma' ñyaksam.

creo ma? ny-daks=am

believe:1SG:PRES PERF 2A:3O-hit=IAM

" "I think you hit her.",

(217) No dyim. Njune'ep naks? dyim.

no $y$-dim nhune?e-p n-daks y-dim

no $3 \mathrm{~S}_{\mathrm{A}}-$ say how-FUT 1A:3O-hit $3 \mathrm{~S}_{\mathrm{A}}-$ say

“"No,” he says. "How could I hit her?” he asks.'

(218) Yomta' eñch dakstaj dyim.

yo:mi?-ta? ?eñč $\emptyset$-daks-tah y-dim

woman-PL NEG $3 \mathrm{~S}_{\mathrm{B}}$-hit-PASS $3 \mathrm{~S}_{\mathrm{A}}$-say

،"Women shouldn't be hit.",

(219) Ke no bes ke kuchay toj? dyim.

que no ves que kučay $\emptyset$-toh y-dim

that no see:2sG:PRES that whip $3 \mathrm{~S}_{\mathrm{B}}-$ hurt $3 \mathrm{~S}_{\mathrm{A}}-$ say

" "Don't you see that the whip hurts?" he says'

(220) Si dyim, pero yä'äp ma' ñyaks dyim.

sí y-dim pero yîip ma? ny-daks y-dim

yes $3 \mathrm{~S}_{\mathrm{A}}$-say but this PERF 2A:3O-hit $3 \mathrm{~S}_{\mathrm{A}}$-say

" "Yes," he says, "but this one, you hit," he says.'

(221) Je'ktoo kki' $\eta$ dyim.

he?kto: ky-ki?n $\quad \mathrm{y}-\mathrm{dim}$

for.this 2A:3O-fear $3 \mathrm{~S}_{\mathrm{A}}-$ say

“"That's why she fears you," he says.'

(222) No dyim, njune'ep ñyaks yoomä'? dyim.

no y-dim nhune?e-p ny-daks yo:mi? y-dim

no $3 \mathrm{~S}_{\mathrm{A}}$-say how-FUT 2A:30-hit woman $3 \mathrm{~S}_{\mathrm{A}}-$ say

“"No," he says, "how could I hit a woman?" he asks.'

(223) Npikta'am mala kostumbre ñyaksta'am yoomä' dyim.

ny-pik-ta?m-D mala costumbre ny-daks-ta?m-D

2A:3O-take-2PL-FUT bad habit 2A:3O-hit-2PL-FUT

yo:mi? y-dim

woman $3 \mathrm{~S}_{\mathrm{A}}-$ say

"“You are going to pick up bad habits, if you hit a woman," he says.'

(224) Njune'ep ñyaks? dyim.

nhune?e-p ny-daks $\quad \mathrm{y}$-dim

how-FUT 2A:3O-hit $3 \mathrm{~S}_{\mathrm{A}}$-say

، "How are you going to hit her?" he says.' 
(225) Yoomä' eñch wää ñyaks dyim.

yo:mi? ?eñč wi: ny-daks y-dim

woman NEG be.able AUx $_{2 \mathrm{~A}}$ 3O-hit $3 \mathrm{~S}_{\mathrm{A}}-$ say

“"You can't hit a woman," he says.'

(226) Jepe' yoswachaj dyim.

hepe? $\emptyset$-yo:s-wat-yah $\quad \mathrm{y}$-dim

this $\quad 3 \mathrm{~S}_{\mathrm{B}}$-work-do-3PL $\quad 3 \mathrm{~S}_{\mathrm{A}}-$ say

، “They work," he says.'

(227) Eñdyee shyun dyima'ap neecheena'.

$\begin{array}{llll}\text { Peñd }{ }^{y} e: & \text { y-sun } & \text { y-dim-a?-p } & \text { ne:če:na? } \\ \text { NEG } & \text { 3A:3O-want } & \text { 3A:3O-say-APPL-FUT } & \text { nothing }\end{array}$

'The (son-in-law) didn't want to tell (the father-in-law) anything

(about how he got the girl to work).'

(228) Ya, pero njune' ma' nwyat? dyim.

ya pero nhune? ma? ny-wat $\mathrm{y}$-dim

ya but how PERF 2A:3O-do $3 \mathrm{~S}_{\mathrm{A}}$-say

"'But how did he do it?" he says (to himself).'

(229) Pero njune' maas? dyim, ñyombaa dyyoswat dyim.

pero nhune? ma:s y-dim ny-jo:mi?-ba:

but how more $3 \mathrm{~S}_{\mathrm{A}}$-say 2PSR-woman-DIM

$$
\begin{array}{ll}
\text { y-yo:s-wat } & \text { y-dim } \\
3 \mathrm{~S}_{\mathrm{A}} \text {-work-do } & 3 \mathrm{~S}_{\mathrm{A}}-\mathrm{say}
\end{array}
$$

" "But how?" he says (to his father-in-law). "Your daughter works," he says.'

(230) Chye'tze'ka'y byumbe pilaat.

y-če? če?-ka?y $\quad \mathrm{y}$-bumbe pila:t

3A:3O-DISTR wash-INTENS 3PSR-all plate

" "She washes all the dishes.'

(231) Däk a'mpuutä'!

dik ?a?m-put-i?

go $_{\text {AUX }}$ see-leave-IMP

" "Go see!",

(232) Ma' täkeñ joktäko'om.

ma? Ø-tikeñ ho:k-tik-o?o-m

PERF $3 \mathrm{~S}_{\mathrm{B}}$-enter smoke-house-LOC-LOC

'He entered the kitchen.'

(233) 'Ya'mkput kya'che' 'yoom.

y-2a?m-k-put $\quad \mathrm{y}$-ka?ače? $\quad \mathrm{y}$-?o:m

3A:3O-look-CINF-leave 3PSR-utensils 3PSR-father

'He looks at the dishes, her father.' 
(234) Ke wää kya'ache' byumbe limpiu echaj!

que wi: $\quad \mathrm{y}$-ka?ače? $\mathrm{y}$-bumbe limpio $\emptyset$-êč-yah

that good 3PSR-utensils 3PSR-all clean $3 \mathrm{~S}_{\mathrm{B}}-\mathrm{be}-3 \mathrm{PL}$

'How beautiful these utensils, everything is clean!'

(235) Byumbe.

y-bumbe

3PSR-all

'Everything.'

(236) Nee dyyojkoy dyye'pkpakyaj njem.

ne: y-dohkoy y-ye?p-k-pak-yah nhem

also 3PSR-pants 3A:3O-hang-CINF-have-3PL there

'He also had his pants hanging there.'

(237) Ya be mä'ät dyim.

ya ve n-birit y-dim

already see:IMP 1PSR-son-in-law $3 \mathrm{~S}_{\mathrm{A}}$-say

' "So you see, my son-in-law" he says.'

(238) ke wää njune' ich dyim.

que wi: nhune? y-?êč $y-d i m$

that good how $3 \mathrm{~S}_{\mathrm{A}}-$ be $3 \mathrm{~S}_{\mathrm{A}}-$ say

“"How nice it is," he says.'

(239) Pero njune' ma' wyat? dyim.

pero nhune? ma? $y$-wat $y$-dim

but how PERF 3A:3O-do 3S -say

" "But how did he do it?" he asks (himself).'

(240) Ma'te eeche', ma' däk chikä.

ma?=te ?êč-e? ma? $\emptyset$-dik y-tik-i

PERF $=$ INC be-DEP PERF $3 \mathrm{~S}_{\mathrm{B}}-$ go 3PSR-house-LOC

'There he was, later he went to his house.'

(241) Ma' du'k chikä, eñch eeche ma' dyima' dyyuuñe'.

$\begin{array}{clllll}\text { ma? } & \emptyset \text {-duPk } & \text { y-tik-i } & \text { Peñč } & \text { Pe:če } & \text { ma? } \\ \text { PERF } & 3 S_{\text {B }} \text {-arrive } & \text { 3PSR-house-LOC } & \text { NEG } & \text { nothing } & \text { PERF } \\ \text { y-dim-a? } & \text { y-du:ñe? } & & & \\ \text { 3A:3O-say-APPL } & \text { 3PSR-woman } & & & \end{array}$

'He arrived at his house and didn't say anything to his wife.'

(242) Nee nje' njumbu' kañcheñ.

ne: nhe? nhumbu? $\emptyset$-kañčeñ

also 3PRO very $3 \mathrm{~S}_{\mathrm{B}}$-lazy

'She was also very lazy.' 
(243) Ma' du'k chikä.

ma? $\emptyset$-du?k $\quad$-tik-i

PERF $3 \mathrm{~S}_{\mathrm{B}}$-arrive 3PSR-house-LOC

'He arrived at his house.'

(244) Ma'te we'eke' ya.

ma?=te $\emptyset$-wê?k-e? ya

PERF $=I N C \quad 3 S_{B}-$ eat - DEP already

'He began to eat.'

(245) Sänkej tụkak jaa.

$\emptyset$-sin-keh tupkak ha:

$3 \mathrm{~S}_{\mathrm{B}}$-sun-appear other day

'He woke up the next day.'

(246) Ma' däk chikä eepä', bi'it.

ma? $\emptyset$-dik y-tik-i Pe:pi? y-birit

PERF $3 \mathrm{~S}_{\mathrm{B}}-\mathrm{go}$ 3PSR-house-LOC other.time 3PSR-son.in.law

'He went back to his son-in-law's house.'

(247) Du'k'yaaywa'k byumbe.

$\emptyset$-du?k y-?a:ywa?k y-bumbe

$3 \mathrm{~S}_{\mathrm{B}}$-arrive 3A:3O-ask 3PSR-all

'He arrived and asked everything.'

(248) Chi' nwyat maan? dyim.

či? ny-wat n-ba:n y-dim

what 2A:3O-do 1PSR-child 3S -say

“"What are you doing, daughter?" he asks.'

(249) Yä'ä k'ech n'oom.

yịił k-?êč n-?o:m

here $1 \mathrm{~S}_{\mathrm{B}}$-be 1PSR-father

،"Here I am, father.",

(250) Wää ij.

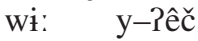

good $3 \mathrm{~S}_{\mathrm{A}}-$ be

، "That's good.'

(251) Bweenu, kdäma'ajä' njunu nwyat ekä nchye'tze'ka'y byumbe? dyim.

bueno k-dim-a?-h-i? nhunu ny-wat Peki

good 3A:1O-say-APPL-LIG-IMP how 2A:3O-do that

$$
\text { ny-ce? фe?-ka?y } \quad \mathrm{y} \text {-bumbe } \mathrm{y} \text {-dim }
$$

2A:3O-DISTR wash-INTENS 3PSR-all $3 \mathrm{~S}_{\mathrm{A}}$-say

" "Good, tell me, how did you do it that you clean everything?", 
(252) No, n'oom dyim, a'y yä'äp pääñche a'yñch nchye'eka'aj,

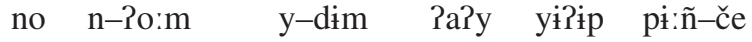

no $1 \mathrm{PSR}-$ father $3 \mathrm{~S}_{\mathrm{A}}$-Say if this man-LIM

$$
\begin{array}{ll}
\text { Pa?y-Reñč } & \text { ny- ce?-ka?ah } \\
\text { if-NEG } & 2 \mathrm{~A}: 3 \mathrm{O}-\text { wash-INTENS:FUT }
\end{array}
$$

“"No, father," she says, "this man, if you don't wash,'

(253) jes dyyu'k dyim,

hes $\quad \mathrm{y}$-durk $\mathrm{y}$-dim

when $3 \mathrm{~S}_{\mathrm{A}}$-arrive $3 \mathrm{~S}_{\mathrm{A}}-$ say

“"when he arrives," she says,'

(254) yä'äp dyim nki'y dyim.
yîip $y$-dim
ny-ki?p
$\mathrm{y}-\mathrm{dim}$
this $3 \mathrm{~S}_{\mathrm{A}}-$ say
2A:3O-have.fear $3 \mathrm{~S}_{\mathrm{A}}$-say
“"this one, he scares you," she says.'

(255) Chi'yoo wyat?

čirjo: $\quad y-w a t$

what.thing 3A:3O-do

" What does he do?",

(256) Juu! dyim. Yä'äp maalu, dyim.

hu: $y$-dim yi?ip malo y-dim

INTER $3 \mathrm{~S}_{\mathrm{A}}$-say this bad $3 \mathrm{~S}_{\mathrm{A}}-$ say

“"Whooh!” she says. "This one's bad," she says.'

(257) Kä'äßä', dyima'wiñ.

$\begin{array}{lll}\text { ki?n-i? } & \text { y-dim-a? } & \text { y-wêñ } \\ \text { have.fear-IMP } & \text { 3A:3O-say-APPL } & 3 \mathrm{~S}_{\mathrm{A}}-\mathrm{REFL}\end{array}$

“ “Fear him, he's fierce.'

(258) Bäkske dyim, dyima' wiñ dyim.

biks-ke y-dim y-dim-a? $\quad$ y-wêñ $\quad y$-dim

much-INT 3S - say 3A:3O-say-APPL $3 \mathrm{~S}_{\mathrm{A}}-\mathrm{REFL} \quad 3 \mathrm{~S}_{\mathrm{A}}-\mathrm{say}$

، "He's very fierce," she said.'

(259) ¿Como?

" How?",

(260) Si, dyima' wiñ.

sí $\quad \mathrm{y}-\mathrm{dim}-\mathrm{a}$ ? $\quad \mathrm{y}$-wêñ

yes 3A:3O-Say-APPL $3 \mathrm{~S}_{\mathrm{A}}-\mathrm{REFL}$

، "Yes, he's fierce.' 
(261) Biiña' chyujku'. Njem chyak juchu wi'k.
y-bêñ-Da?
y-tuh-ku?
nhem $\quad \mathrm{y}-\mathrm{cak}$
3A:3O-come-CAUS 3PSR-shoot-INSTR.NOM
there 3A:3O-leave

huč $\quad$ Pu $\quad \mathrm{y}$-wê?k

where IMPF $3 \mathrm{~S}_{\mathrm{A}}$-eat

" "He comes with his gun. There he leaves it where he eats.

(262) Njem iicha'.

nhem $\mathrm{y}-$ Pêč-Da?

there 3A:3O-be-CAUS

“ "There he has it.'

(263) 'Yaāwejäpaapä' chyujka'ap.

y-?a:yweh-a?-pa:=pi? $\quad$ y-tuh-ka?-p

3A:3O-shout-APPL-NOM=REL $3 \mathrm{~A}: 3 \mathrm{O}-$-shoot-INTENS-FUT

، "Whoever shouts at him, he'll shoot.'

(264) Wyanäpaapä' chyujka'ap.

$\mathrm{y}-$ wan-a?-pa:=pi? $\quad \mathrm{y}-\mathrm{tuh}-\mathrm{ka}$ ? $-\mathrm{p}$

3A:3O-sing-APPL-NOM=REL $3 \mathrm{~A}: 3 \mathrm{O}-$-shoot-INTENS-FUT

“"Whoever sings to him, he'll shoot.'

(265) Ya be dyim, jepe' jaa dyim, ma' du'k wokde' ich dyim.

ya ve $y$-dim hepe? ha: y-dim ma?

already see:IMP $3 \mathrm{~S}_{\mathrm{A}}-$ say this day $3 \mathrm{~S}_{\mathrm{A}}$-say PERF

$$
\begin{array}{llll}
\emptyset \text {-duPk } & \emptyset \text {-wok-de? } & \text { y-Rêč } & \text { y-dim } \\
3 \mathrm{~S}_{\mathrm{B}} \text {-arrive } & 3 \mathrm{~S}_{\mathrm{B}} \text {-angry-STV } & 3 \mathrm{~S}_{\mathrm{A}} \text {-be } & 3 \mathrm{~S}_{\mathrm{A}}-\text { say }
\end{array}
$$

" "You see," she says, "that day," she says," he came home very angry," she says.'

(266) Eñdyee shyun ootätajp.

$$
\begin{array}{lll}
\text { Peñd } \mathrm{y}_{\mathrm{e}}: & \mathrm{y}-\text { sun } & \emptyset-\text { - o:ta?-tah-p } \\
\text { NEG } & \text { 3A:3O-want } & 3 \mathrm{~S}_{\mathrm{B}} \text {-speak-PASS-FUT }
\end{array}
$$

“'He didn't want to be spoken to.'

(267) Eñdyee shyun aạwejätajp.

$$
\begin{array}{lll}
\text { Peñdye: } & \text { y-sun } & \emptyset-\text { - } a: \text { nweh-a?-tah-p } \\
\text { NEG } & \text { 3A:3O-want } & 3 \mathrm{~S}_{\mathrm{B}} \text {-shout-APPL-PASS-FUT }
\end{array}
$$

“'He didn't want to be shouted at.'

(268) Eñdyee wanätajp.

?eñdye: $\emptyset$-wan-a?-tah-p

NEG $\quad 3 \mathrm{~S}_{\mathrm{B}}$-sing-APPL-PASS-FUT

“"He didn't want to be sung to.' 
(269) Eñdyee shyun neecheena'.

$\begin{array}{lll}\text { Peñdye: } & \text { y-sun } & \text { ne:če:na? } \\ \text { NEG } & 3 \mathrm{~A}: 3 \mathrm{O}-\text { want } & \text { nothing }\end{array}$

“"He didn't want anything.'

(270) Poko chyujka'ap.

porque $\quad \mathrm{y}$-tuy-ka?-p

because 3A:3O-shoot-INT-FUT

،"Because he was going to kill (that person).'

(271) Pobre kaydya'a dyim ma' wan dyim,

pobre gallo-da?a y-dim ma? Ø-wan y-dim

poor rooster-AUM $3 \mathrm{~S}_{\mathrm{A}}$-say PERF $3 \mathrm{~S}_{\mathrm{B}}-\operatorname{sing} 3 \mathrm{~S}_{\mathrm{A}}$-say

“"The poor rooster sang," she said,'

(272) i ma' chyujka' dyim.

?i ma? y-tuh-ka? y-dim

and PERF 3A:3O-shoot-INT 3S -say

" "and he shot it," she says.

(273) Aa dyim, wää ich dyim.

a: $\quad y-\operatorname{dim} \quad$ wi: $\quad y-$-rěč $\quad y$-dim

aa $3 \mathrm{~S}_{\mathrm{A}}$-say good $3 \mathrm{~S}_{\mathrm{A}}$-be $3 \mathrm{~S}_{\mathrm{A}}$-say

“"Ah," he says, "it's fine," he says.

(274) Aa, wää ich dyim.

a: wi: $y$-?êč $\quad y-d i m$

aa good $3 \mathrm{~S}_{\mathrm{A}}$-be $3 \mathrm{~S}_{\mathrm{A}}$-say

، “Ah, it's fine," he says.'

(275) Si dyim.

sí $\quad \mathrm{y}$-dim

yes $3 \mathrm{~S}_{\mathrm{A}}-$ say

“"Yes," she says.'

(276) Pobre kaydya'a dyim.

pobre gallo-da?a y-dim

poor rooster-AUGM $3 \mathrm{~S}_{\mathrm{A}}$-say

“"The poor rooster," she says.

(277) Pey'aap dyim, ma' chyujka', dyim,

pollo-?a:p y-dim ma? y-tuh-ka? y-dim

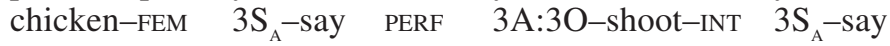

“"The poor chicken he shot,' 
(278) poko ma'che wan.

poco ma?-če $\emptyset$-wan

because PERF-LIM $3 \mathrm{~S}_{\mathrm{B}}-$ sing

" "Because he sang.'

(279) Eñdyee shyun wanätajp dyim.

?eñdye: y-sun $\quad \emptyset$-wan-a?-tah-p $\quad$ y-dim

NEG 3A:3O-want $3 \mathrm{~S}_{\mathrm{B}}$-sing-APPL-PASS-FUT $3 \mathrm{~S}_{\mathrm{A}}$-say

“"He didn't want to be sung to," she says.'

(280) Jes dyim, ma' chyujka' dyim.

$\begin{array}{lllll}\text { hes } & \text { y-dim } & \text { ma? } & \text { y-tuh-ka? } & \text { y-dim } \\ \text { now } & 3 \mathrm{~S}_{\mathrm{A}}-\text { say } & \text { PERF } & \text { 3A:3O-shoot-INT } & \text { 3S }- \text {-say }\end{array}$

“"Now, he'll shoot it," she says.'

(281) Aa dyim, wää ich dyim, maan dyim.

a: y-dim wi: y-?êč y-dim n-ba:n y-dim

ah $3 \mathrm{~S}_{\mathrm{A}}$-say good $3 \mathrm{~S}_{\mathrm{A}}$-be $3 \mathrm{~S}_{\mathrm{A}}$-say $1 \mathrm{PSR}-$ child $3 \mathrm{~S}_{\mathrm{A}}$-say

“"Ah," he says, "that's fine, daughter," he says.'

(282) Yoswaate'! dyim.

$\emptyset$-yo:s-wat-e? $\quad \mathrm{y}$-dim

$3 \mathrm{~S}_{\mathrm{B}}$-work-do-opT $3 \mathrm{~S}_{\mathrm{A}}-$ say

“"She works!" he says.'

(283) Entonse ma' put da'anpa'kshdya'a.

entonces ma? $\emptyset$-put da?aypa?kš-da?a

then PERF $3 \mathrm{~S}_{\mathrm{B}}$-exit husband-AUGM

'Then the husband left.' 3

(284) Ma' däk chikä.

ma? $\emptyset$-dik $\quad \mathrm{y}-\mathrm{tik}-\mathrm{i}$

PERF $3 \mathrm{~S}_{\mathrm{B}}$-go 3PSR-house-LOC

'He left the house.'

(285) Du'k chikä njem.

$\emptyset$-du?k y-tik-i nhem

$3 \mathrm{~S}_{\mathrm{B}}$-arrive 3PSR-house-LOC there

'He arrived there at his house.'

(286) Nee nje' ma' jiikbatzkak chyujku'.

ne: nhe? ma? y-hi:k-baø-kak y-tuh-ku?

also 3PRO PERF 3A:3O-pull-take-REPET 3PSR-shoot-INSTR.NOM

'He also grabbed his gun.'

3 The word da?aypa?kf is composed of the root da?ay 'husband' and the unidentified root pa?kš. In this line, the word refers to the father-in-law. 
(287) Byeja meeña'ajä' k'we'kp! dyim.

vieja $\emptyset$-mêñ-Da?-h-i? $\quad \mathrm{k}$-wê?k-p $\quad \mathrm{y}$-dim

old.woman $3 \mathrm{~S}_{\mathrm{B}}$-come-CAUS-LIG-IMP $\quad 1 \mathrm{~S}_{\mathrm{B}}$-eat-FUT $3 \mathrm{~S}_{\mathrm{A}}$-say

“"Wife, bring it, I'm going to eat!" he says.'

(288) Asta yä'äp jaa dyim, eñdyee nsun ee k'aanweja'ap, dyim.

hasta yi?ip ha: $y$-dim Peñdye: n-sun

until this day $3 \mathrm{~S}_{\mathrm{A}}$-say NEG 1A:30-want

$$
\begin{array}{lll}
\text { Pe: } & \text { k-Pa:yweh-a?-p } & y-d i m \\
\text { someone } & \text { 3A:1O-shout-APPL-Fut } & 3 \mathrm{~S}_{\mathrm{A}} \text {-say }
\end{array}
$$

“'As of today, I don't want anyone to shout at me," he says.'

(289) Aanwejpaapä' dyim, nyaka'ap.

$\emptyset-? a: y w e h-p a:=p a ̈ ? \quad$ y-dim n-yaka?-p

$3 \mathrm{~S}_{\mathrm{B}}$-shout-NOM=REL $3 \mathrm{~S}_{\mathrm{A}}$-say $1 \mathrm{~A}: 3 \mathrm{O}-$ kill-FUT

“"Whoever shouts," he says, "I will kill.'

(290) K'ootäpaapä' nyaka'ap.

k-Po:ta?-pa:=pi? $\quad$ n-yaka?-p

3A:1O-speak-NOM=REL $\quad$ 1A:3O-kill-FUT

'“Whoever speaks to me, I will kill.'

(291) K'wanäpaapä' nyaka'ap nee nje'.

k-wan-a?-pa:=pi? $\quad$ n-yaka?-p ne: nhe?

3A:1O-sing-APPL-NOM=REL 1A:3O-kill-FUT also 3PRO

“"Whoever sings, I will also kill.",

(292) I dyyuuñe' eñdye ' yoota', upche byatän.

Pi y-du:ñe? Peñdye y-Po:ta? Pupa-če

and 3PSR-woman NEG 3A:3O-speak now-LIM

y-batin

3A:3O-listen

'And his woman did not speak to him, she just listened.'

(293) Jesa byatän ma' aanwejksaj tum burrubaa.

hesa y-batin ma? $\emptyset$-?a:ywey-ksah tum burro-ba:

when 3A:3O-listen PERF $3 \mathrm{~S}_{\mathrm{B}}$-shout-DF one donkey-DIM

'When he listened, a donkey brayed.'

(294) 'Yaanwej ich.

y-?a:yweh y-?êč

$3 \mathrm{~S}_{\mathrm{A}}$-shout $3 \mathrm{~S}_{\mathrm{A}}-$ be

'It was there braying.'

(295) Jnnk jnnk jnnk.

'Hee haw hee haw hee haw.' 
(296) ¡Bueno!” Y, “ ¡Hijo de la chingada! dyim.

“"Good!” And, “Ay, son of a bitch!" he says.'

(297) Yä'äp bäskbäskburrtukuda'a, k'wat insultar dyim.

yiłip bis $\sim$ bis-k-burro-tuku?-da?a k-wat

here DISTR disgust-CINF-donkey-old-AUGM 3A:1O-do

insultar $\mathrm{y}-\mathrm{dim}$

insult $\quad 3 \mathrm{~S}_{\mathrm{A}}-$ say

“"This disgusting donkey, he is insulting me," he says.'

(298) Jiikput chyujku', njem chyujka'ajam.

y-hi:k-put y-tuh-ku? nhem

3A:3O-pull-exit 3PSR-shoot-INSTR.NOM there

y-tuh-ka?-h=am

3A:3O-shoot-INTENS-LIG=IAM

'He took out his pistol (and) there he shot it.'

(299) Chyujka'.

y-tuh-ka?

3A:3O-shoot-INTENS

'He shot it.'

(300) Ma'y chyujka', ma'te we'eke', ma' yaj.

ma?-ya y-tuh-ka? ma?=te $\emptyset$-wê?k-e?

PERF-already 3A:3O-shoot-INTENS PERF=INC $3 \mathrm{~S}_{\mathrm{B}}$-eat-DEP

ma? $\emptyset$-yah

PERF $3 \mathrm{~S}_{\mathrm{B}}-$ finish

'When he shot it, he began to eat, he finished.'

(301) Ma' n'isham?

ma? ny-?êšs=am

PERF 2A:3O-See $=$ IAM

" "You see?",

(302) K'aaฤwejäpaapä' ntujka'ap.

k-?a:yweh-a?-pa:=pi? n-tuh-ka?-p

3A:1O-shout-APPL-NOM=REL $1 \mathrm{~A}: 3 \mathrm{O}-$-shoot-INT-FUT

'“Whoever shouts at me, I will kill.'

(303) K'ootäpaapä' ntujka'ap.

k-Po:ta?-pa:=pi? n-tuh-ka?-p

3A:1O-speak-NOM=REL $\quad 1 \mathrm{~A}: 3 \mathrm{O}-$-shoot-INT-FUT

“"Whoever speaks to me, I will shoot.", 
(304) Dyyuuñe' 'yoota',

y-du:ñe? $\quad$-1o:ta?

3PSR-woman 3A:3O-speak

'His wife told him,'

(305) Bich njyaam.

bič ny-ha:m

2PRO 2A:3O-know

" YYou know best.",

(306) I yoompu' burru ma' däk boy jiikpokeñ.

?i $y-$-o:m-pu? burro ma? dik

and 3PSR-owner-REL donkey PERF $\mathrm{gO}_{\text {AUX }}$

boy $\quad \mathrm{y}$-hi:k-pokeñ

go.and.return $_{\text {AUX }}:$ PERF $3 \mathrm{~A}: 3 \mathrm{O}-$ pull-roll

'And the donkey's owner went to file charges.'

(307) Däk k'o'otja'ajä' yä'äp pääñ dyim. Ma' ktujkäja' murru.

dik k-Po:ta?-h-a?-h-i? yi?ip pi: ñ

go $_{\mathrm{AUX}}$ 3A:1O-speak-LIG-APPL-LIG-IMP this man

y-dim ma? k-tuh-ka?-h-a? n-burro

$3 \mathrm{~S}_{\mathrm{A}}$-say PERF 3A:1O-shoot-INT-LIG-APPL 1PSR-donkey

“"Go speak to this man for me!" he says. "He shot my donkey.",

(308) Che'ka'y? dyim.

če?ka?y y-dim

because $3 \mathrm{~S}_{\mathrm{A}}-$ say

“"Why?” (the police) say.'

(309) Pwe eñch n'eshp che'ka'y.

pues Reñč $n-$-?ěš-p če?ka?y

well NEG 1A:3O-know-FuT why

“'Well, I don't know why.'

(310) Upchi'k jyakdas murrubaa dyim.

Pupa-čîk y-hak-das n-burro-ba: y-dim

now-PAST $3 \mathrm{~S}_{\mathrm{A}}-$ pass-pass 1 PSR-donkey-DIM $3 \mathrm{~S}_{\mathrm{A}}$-say

“'My donkey was passing by," he says.'

(311) Njem ma' chyujka' dyim.

nhem ma? y-tuh-ka?

there PERF 3A:3O-shoot-INTENS 3S $\mathrm{A}_{\mathrm{A}}$-say

“"There he shot him.", 
(312) Aa kabron dyim.

a: cabrón y-dim

INTER jerk $3 \mathrm{~S}_{\mathrm{A}}$-say

“"Ah, the jerk," he says.'

(313) Pwes däksä' päkta'amä'.

pues diks-i? $\emptyset$-pik-ta?m-i?

well go-IMP $3 \mathrm{~S}_{\mathrm{B}}$-bring-2PL-IMP

“"Well, go, go get him.",

(314) Däksä' däk puuta'ajä' chiko'o.

diks-i? dik $\emptyset$-put-Da?-h-i? $\quad$-tik-o?o

go-IMP $\quad$ go $_{\text {AUX }} \quad 3 \mathrm{~S}_{\mathrm{B}}$-exit-CAUS-LIG-IMP 3PSR-house-LOC

“"Go get him from his house!'

(315) Däk puuta'ajä', meeña'ajä'.

dik put-Da?-h-i? mêñ-Da?-h-i?

go $_{\text {AUX }}$ exit-CAUS-LIG-IMP come-CAUS-LIG-IMP

" "Go get him, bring him!",

(316) Chiwä dyyaka' tum animal?

čiwi y-yaka? tum animal

why 3A:3O-kill one animal

" "Why does he kill an animal?",

(317) Chi' 'yoota' si animal eñch n'ishp?

či? y-Po:ta? si animal ?eñč y-?êš-p

what 3A:3O-speak if animal NEG 3A:3O-know-FUT

" "What's it going to say if the animal doesn't know?",

(318) Ma' meñaj pääñ.

ma? $\emptyset$-mêñ-yah pi:ñ

PERF $3 \mathrm{~S}_{\mathrm{B}}-$ come-3PL man

'The men came.'

(319) Du'kyaj taaksä'y.

$\emptyset$-du?k-yah ta:ksi?y

$3 \mathrm{~S}_{\mathrm{B}}-$ arrive-3PL police

'The police came.'

(320) Por fabor dyim, bich ñiy Julaanu?

por_favor $\mathrm{y}$-dim bič ny-diy Julano

please $3 \mathrm{~S}_{\mathrm{A}}$-say 2PRO 2PSR-name Julano

" "Please," (the policeman) says, "Is your name is Julano?",

(321) Dyim, si dyim.

y-dim sí $\quad \mathrm{y}-\operatorname{dim}$

$3 \mathrm{~S}_{\mathrm{A}}$-say yes $3 \mathrm{~S}_{\mathrm{A}}-$ say

'He responds, "Yes,' he says.' 
(322) Tej! dyim.

teh $\quad \mathrm{y}-\mathrm{dim}$

let's.go $3 \mathrm{~S}_{\mathrm{A}}-$ say

“"Let's go!" he says.'

(323) Pero che'ka'y?

pero čeka?y

but why

"'But why?",

(324) Tej, ma'y knäma'!

teh ma?=ya kn-dim-a?

let's.go PERF=already 1A:2O-say-APPL

، "Let's go, I tell you!'

(325) A'yñch kpyutp, näämä'!

?a?y-?eñč ky-put-p nim-i?

if-NEG $2 \mathrm{~S}_{\mathrm{B}}$-exit-FUT say-IMP

" "If you're not going to leave, say so!",

(326) Njem jiikpuchaj chiko'o.

nhem y-hi:k-put-yah y-tik-o?o

there 3A:3O-pull-exit-3PL 3PSR-house-from

'There they drag him outside the house.'

(327) Ma' dyiiksäyajam.

ma? y-diks-Da?-yah=am

PERF 3A:3O-go-CAUS-3PL=IAM

'They carried him off.'

(328) Pyatzka'myaj dakshäko'o.

y-pađ̌-ka?m-yah dakšik-o?o

3A:3O-throw-between-3PL jail-LOC

'They throw him in jail.'

(329) Pyatzka'myaj.

y-paç-kam-yah

3A:3O-throw-enter-3PL

'They throw him inside.'

(330) Ma' däk preeso.

ma? $\emptyset$-dik preso

PERF $3 \mathrm{~S}_{\mathrm{B}}-\mathrm{go}$ imprisoned

'He's imprisoned.'

(331) Ahora sí.

'Now, yes.' 
(332) Jeksh si ma' täkeñ.

hekš sí ma? $\emptyset$-tikeñ

now yes PERF $3 \mathrm{~S}_{\mathrm{B}}$-enter

'Now, yes, he went in.'

(333) Tuykak jaa ma' säykej.

tunkak ha: ma? $\emptyset$-sin-keh

other day PERF $3 \mathrm{~S}_{\mathrm{B}}$-sun-appear

'The next day he woke up.'

(334) Chi'yoo nä'äksja'? dyim.

čîyo: n-diks-Da?-h-a? y-dim

what 1A:3O-go-CAUS-LIG-APPL $3 \mathrm{~S}_{\mathrm{A}}$-say

“"What do I owe?" he says.'

(335) Njem k'yoota' tum pääñ dyim.

nhem ky-?o:ta? tum pi:ñ y-dim

there 2A:30-speak one man $3 \mathrm{~S}_{\mathrm{A}}-$ say

“"There's a man (who wants) to speak to you.'

(336) Ätz eñch n'esh chi' ma' nwyata' dyim.

Piф Reñč n-?êš či? ma? ny-wat-a?

1PRO NEG 1A:3O-know what PERF 2A:3O-do-APPL

$$
\mathrm{y}-\operatorname{dim}
$$

$3 \mathrm{~S}_{\mathrm{A}}-$ say

“"I don't know what you did to him," he says.'

(337) Dyim ma'm ñya'akja' byurru.

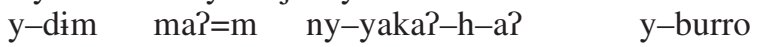

$3 \mathrm{~S}_{\mathrm{A}}$-say $\quad$ PERF=REP 2A:3O-kill-LIG-APPL 3PSR-donkey

“"He says you killed his donkey.",

(338) Aa dyim.

a: $\quad y-\operatorname{dim}$

ah $3 \mathrm{~S}_{\mathrm{A}}-\mathrm{say}$

“"Ah," he says.'

(339) Sta byen dyim.

está bien $\mathrm{y}$-dim

be:3sG:PRES fine $3 \mathrm{~S}_{\mathrm{A}}$-say

“"That's fine," he says.'

(340) Ma' däk njem.

ma? $\emptyset$-dik nhem

PERF $\quad 3 \mathrm{~S}_{\mathrm{B}}-\mathrm{go}$ there

'There he went.' 
(341) Chi' nshyun kopkpääñ? dyim.

či? ny-sun kopik-pi:ñ y-dim

what 2A:3O-want head-man $3 \mathrm{~S}_{\mathrm{A}}$-say

'“What do you want, Mr. Mayor?" he says.'

(342) Pwes ätz eñch eeche nsun.

pues ?iф Reñč Re:če n-sun

well 1PRO NEG nothing 1A:3O-want

“"Well, I don't want anything.",

(343) Yä'ä ech yä'äp pääñ dyim,

yîi $\emptyset$-?êč yirip pi:̃̃ y-dim

here $3 \mathrm{~S}_{\mathrm{B}}$-be this man $3 \mathrm{~S}_{\mathrm{A}}$-say

“"There's a man here," he says,'

(344) dyim bich ma'm ñya'akja' dyim, byurru.

$\mathrm{y}-\mathrm{dim} \quad$ bič $\quad \mathrm{ma}=\mathrm{m} \quad$ ny-yaka?-h-a? $\mathrm{y}-\mathrm{dim}$

$3 \mathrm{~S}_{\mathrm{A}}$-say 2 PRO PERF=REP $2 \mathrm{~A}: 3 \mathrm{O}-$ kill-LIG-APPL $3 \mathrm{~S}_{\mathrm{A}}$-say

y-burro

3PSR-donkey

"'he says that you killed his donkey.'

(345) Njem bich ma' ñya'akja' byurru.

nhem bič ma? ny-yaka?-h-a? y-burro

there 2PRO PERF 2A:3O-kill-LIG-APPL 3PSR-donkey

“"You killed his donkey there.",

(346) Dyim, si dyim, ätza' nyaka' dyim.

y-dim sí y-dim ił $\not=m a ? \quad n$-yaka? y-dim

$3 \mathrm{~S}_{\mathrm{A}}$-say yes $3 \mathrm{~S}_{\mathrm{A}}$-say 1PRO=PERF 1A:3O-kill $3 \mathrm{~S}_{\mathrm{A}}$-say

'He says, "Yes," he says, "I killed it," he says.'

(347) Pwe, jeksh ñyojp.

pues hekš ny-yoh-p

well now 2A:3O-pay-FuT

“"Well, now you're going to pay.'

(348) Si no yä'ä eñch kpyutp dyim.

si no yi?i ?eñc ky-put-p y-dim

si no here NEG $2 \mathrm{~S}_{\mathrm{B}}$-exit-FuT $3 \mathrm{~S}_{\mathrm{A}}$-say

“"If not, you're not going to get out of here," he says.'

(349) Yä'ä kntzakta'am dyim, wäsna' tukna' amche.

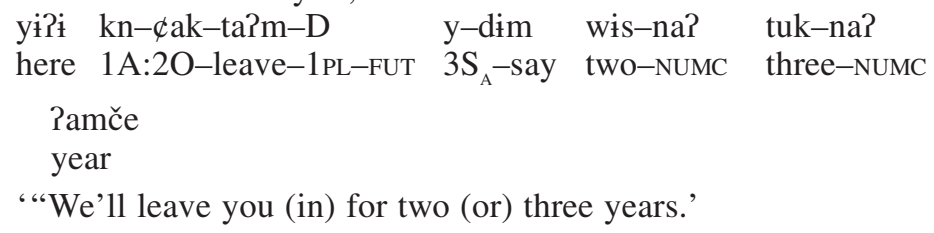


(350) Kchi'ij yä'äm dyim, pwe ñyojp burru dyim.

ky- $\not \dot{i}$ ith $\quad$ yi?i-m $\mathrm{y}$-dim pues ny-yoh-p

$2 \mathrm{~S}_{\mathrm{B}}$-stay:Fut here-Loc $3 \mathrm{~S}_{\mathrm{A}}$-say well 2A:3O-pay-FuT

burro $\mathrm{y}-\mathrm{dim}$

donkey $3 \mathrm{~S}_{\mathrm{A}}-$ say

" "You're going to stay here, (or) well, you're going to pay him for his donkey," he says.'

(351) Che'ka'y ma' ñyaka'?

če?ka?y ma? ny-yaka?

why PERF 2A:3O-kill

“"Why did you kill it?'

(352) Chi' ma'k'wyata'?

či? ma? ky-wat-a?

what PERF 2A:3O-do-APPL

"What did he do to you?",

(353) Si dyim, señor, eñch eeche ma' k'wata' dyim.

sí y-dim señor ?eñč ?e:če ma? k-wat-a?

yes $3 \mathrm{~S}_{\mathrm{A}}$-say man NEG nothing PERF 3A:1O-do-APPL

$$
\mathrm{y}-\mathrm{dim}
$$

$3 \mathrm{~S}_{\mathrm{A}}$-say

“"Yes," he says. "Sir, he didn't do anything to me.'

(354) Pääñ eñch eeche ma' k'wata'.

pi:ñ Peñ̌ Pe:če ma? k-wat-a?

man NEG nothing PERF 3A:1O-do-APPL

“'Sir, he didn't do anything to me.'

(355) Wäänää burru upchi'k jyakdas.

wi:ni: burro ?upa=čîk $\mathrm{y}$-hak-das

honest donkey now=PAST $3 \mathrm{~S}_{\mathrm{A}}$-pass - pass

“'In all honesty, he was just passing by.'

(356) Ma' ntujka' dyim.

ma? n-tuh-ka? y-dim

PERF 1A:3O-shoot-INTENS 3S - -say

" "I shot him," he said.'

(357) Pwe, jeksh dyim, ñyojp.

pues hekš $y$-dim ny-yoh-p

well now $3 \mathrm{~S}_{\mathrm{A}}$-say 2A:3O-pay-FuT

" "Well, now you're going to pay.",

(358) Si dyim. Nyojp pwe.

sí $\quad \mathrm{y}$-dim $\mathrm{n}-\mathrm{yoh}-\mathrm{p} \quad$ pues

yes $3 \mathrm{~S}_{\mathrm{A}}$-say 1A:3O-pay-Fut well

“"Yes," he says, "Well, I'm going to pay.' 
(359) Däk näma'ajä' mi byeja dyim,

dik $\emptyset$ nim-a?-h-i?

mi vieja $\quad \mathrm{y}-\mathrm{dim}$

toward.there $3 \mathrm{~S}_{\mathrm{B}}$-Say-APPL-LIG-IMP my old.woman $3 \mathrm{~S}_{\mathrm{A}}$-say

“"Go tell my wife!'

(360) ke biiña'aje' tumeeñ dyim.

que $\mathrm{y}$-bêñ $-\mathrm{a}$ ?-h-e? tume: $\tilde{n}$ y-dim

that 3A:3O-come-APPL-LIG-DEP money $3 \mathrm{~S}_{\mathrm{A}}$-say

“"to bring money," he says.'

(361) Ma' däk.

ma? $\emptyset$-dik

PERF $3 \mathrm{~S}_{\mathrm{B}}-\mathrm{go}$

'He went.'

(362) Däksä', däku'm a'amä' ñya'an.

diks-i? dik=u?m $\emptyset-? a ? m-i ? \quad$ ny-da?a

go-IMP $\quad$ gO $_{\mathrm{AUX}}=\mathrm{REP} \quad 3 \mathrm{~S}_{\mathrm{B}}$-see-IMP $2 \mathrm{PSR}-$ husband

“"Go, go see your husband!" (his wife is told).'

(363) Däksa'ajä' tumeeñ.

diks-Da?-h-i? tume: $\tilde{n}$

go-CAUS-LIG-IMP money

“"Bring money!",

(364) Eñdyee knäma?' dyim.

Peñdye: kn-dim-a? $\quad y$-dim

NEG 1A:2O-say-APPL $3 \mathrm{~S}_{\mathrm{A}}$-say

“"Didn't I tell you?" she says.'

(365) Jyaam wiñ dyima' wiñ.

y-ha:m $\quad y$-wêñ $\quad y$-dim-a? $\quad y$-wêñ

3A:3O-feel $3 \mathrm{~S}_{\mathrm{A}}-\mathrm{REFL} \quad 3 \mathrm{~A}: 3 \mathrm{O}-\mathrm{Say}-\mathrm{APPL} \quad 3 \mathrm{~S}_{\mathrm{A}}$-SELF

“"He feels very brave," she says.'

(366) Dyyachknäma' wiñ, ma' du'k nte'keyaj.

y-dač-ke-nim-a? y-wêñ ma? $\emptyset$-du?k

3A:3O-very-INTENS-SAY-APPL $3 \mathrm{~S}_{\mathrm{A}}$-REFL PERF $3 \mathrm{~S}_{\mathrm{B}}$-arrive

nte?keyah

yesterday

“"He felt very brave (when) he arrived yesterday.'

(367) Dyyachknäma' wiñ dyim.

y-dač-ke-nim-a? $\quad y$-wêñ $\quad y$-dim

3A:3O-very-INTENS-Say-APPL $3 \mathrm{~S}_{\mathrm{A}}-\mathrm{REFL} \quad 3 \mathrm{~S}_{\mathrm{A}}$-say

'“He felt very brave.' 
(368) Ma'm chyujkäkak tum burru.

$\begin{array}{llll}\mathrm{ma} P=\mathrm{m} & \mathrm{y}-\mathrm{tuh}-\mathrm{ka} \text { - }-\mathrm{kak} & \text { tum } & \text { burro } \\ \text { PERF }=\text { REP } & \text { 3A:3O-shoot-INTENS-REPET } & \text { one } & \text { donkey }\end{array}$

“"They say he shot a donkey.'

(369) Chi'sh wyatäkak?

če?-iš $\quad y-w a t-a ?-k a k$

what-FUT 3A:3O-do-APPL-REPET

"“What was it going to do to him?",

(370) Eñdye k'yoota', jepe' jyakdas dyim.

Peñdye ky-?o:ta? hepe? y-hak-das y-dim

NEG 2A:3O-speak this $3 \mathrm{~S}_{\mathrm{A}}$-pass-pass $3 \mathrm{~S}_{\mathrm{A}}$-say

“"It didn't say anything to you, it was (just) passing by," she says.'

(371) Pero bich ma' nchyujka'dyim.

pero bič ma? ny-tuh-ka? y-dim

but 2PRO PERF 2A:3O-shoot-INTENS 3S -say

“"But you shot it," she says.'

(372) Ma' du'k njem.

ma? $\emptyset$-du?k nhem

PERF $3 \mathrm{~S}_{\mathrm{B}}$-arrive there

'She arrives there.'

(373) Entonse chi' nwyat? dyim.

entonces či? ny-wat $y$-dim

then what 2A:3O-do 3S -say

'“What are you going to do?" she says.'

(374) Pwe yojä' burru dyim, ma'pu' ntujka' dyim.

pues yoh-i? burro y-dim ma?=pu?

well pay-IMP donkey $3 \mathrm{~S}_{\mathrm{A}}$-say PERF=REL

$$
\begin{array}{ll}
\text { n-tuh-ka? } & \mathrm{y} \text {-dim } \\
\text { 1A:3O-shoot-INTENS } & 3 \mathrm{~S}_{\mathrm{A}} \text {-say }
\end{array}
$$

“"Well, pay for the donkey that I shot!" he says.'

(375) Eñdye knäma'? dyim.

?eñdye kn-dim-a? $\quad y-d i m$

NEG 1A:2O-say-APPL $3 \mathrm{~S}_{\mathrm{A}}$-say

“"Didn't I tell you?" she says.'

(376) Kpya'da'anma bich dyim.

ky-pa?-da?a $=m a \quad$ bič $\quad y-d i m$

$2 \mathrm{~S}_{\mathrm{B}}$-coward-old=IAM 2 PRO $3 \mathrm{~S}_{\mathrm{A}}$-say

"'You old coward," she says.' 
(377) Jeksh yojä' dyim.

hekš yoh-î? y-dim

now pay-IMP $3 \mathrm{~S}_{\mathrm{A}}$-say

“"Now you pay!"

(378) Yä'ä ech.

yîił $\emptyset-$-?êč

here $3 S_{\mathrm{B}}-$ be

" "Here it is.",

(379) Ma' dyyoj.

ma? y-yoh

PERF 3A:3O-pay

'He pays.'

(380) Tej! dyim.

teh $\quad \mathrm{y}-\mathrm{dim}$

let's.go $3 \mathrm{~S}_{\mathrm{A}}-\mathrm{say}$

“"Let's go!" she says.'

(381) Eepä' watkaakä' dyim, para wa'pa nwi'k yä'äp pooy dyim.

?e:pi? wat-kak-i? y-dim para wa?-pa

again do-REPET-IMP $3 \mathrm{~S}_{\mathrm{A}}$-say for IMP:NEG-FUT

ny-wê?k yîip po:y $\mathrm{y}-\mathrm{dim}$

$2 \mathrm{~S}_{\mathrm{A}}$-eat this month $3 \mathrm{~S}_{\mathrm{A}}-$ say

'“Do it again so you don't eat this month!" she says.'

(382) Che' nesesidad yaj nchyumeeñ? dyim.

če? necesidad $\emptyset$-yah ny-tume:ñ y-dim

what need $\quad 3 \mathrm{~S}_{\mathrm{B}}-$ finish 2PSR-money $3 \mathrm{~S}_{\mathrm{A}}$-say

“"Why do you need your money finished off?" she asks.'

(383) Dyim, jepe' ätzchi'k nshyun knwyatp njunu ma' wattaj ñyombaa? dyim.

y-dim hepe? ii $\phi=\phi i ? k$ ny-sun kny-wat-p

$3 \mathrm{~S}_{\mathrm{A}}$-say this 1PRO=PAST 2A:3O-want 2A:1O-do-Fut

nhunu ma? $\emptyset$-wat-tah ny-yo:mi?-ba: $\mathrm{y}$-dim

hOW PERF $3 \mathrm{~S}_{\mathrm{B}}$-do-PASS 2PSR-woman-DIM $3 \mathrm{~S}_{\mathrm{A}}$-say

"“You wanted to do to me what was done to your daughter?" she asks.'

(384) Pwe, ätz eñch knwyatp je'ye'e, dyim.

pues ?iф ?eñč kny-wat-p he?ye?e y-dim

well 1PRO NEG 2A:1O-do-FUT like $3 \mathrm{~S}_{\mathrm{A}}$-say

“"Well, you're not going to do that to me," she says.' 
(385) Ya kduuñma. I a'y da'anma tum kuy, njusna' däk ñyumwaate' dyim wo'kskä'da'ap,

ya k-du:ñe?=ma ?i ?a?y da?ay=ma tum kuy already $1 \mathrm{~S}_{\mathrm{B}}-\mathrm{old}=\mathrm{IAM}$ and if old=IAM one stick

nhu:sa-na? dik ny-du:mu?-wat-e? $\quad$-dim

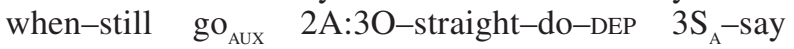

$\emptyset$-wo?ks-ki?da?a-pi?

$3 \mathrm{~S}_{\mathrm{B}}$-crooked-ADJZ-REL

" "I am old already. And if a stick is old, how are you going to straighten what's crooked,'

(386) a'y wächeda'ajam?

?a?y $\emptyset$-wiče?-da?a-h=am

if $\quad 3 \mathrm{~S}_{\mathrm{B}}$-big-AUGM-LIG=IAM

“"if it's already big?'

(387) Jepe' nje' jeschi'k jisäbaa jeschi'k ñyumwat.

hepe? nhe? hes=či?k y-hisi-ba: hes=čirk

this 3PRO when=PAST 3PSR-small-DIM when=PAST

ny-du:mu?-wat

2A:3O-straighten-do

'“This, when it was small is when you should have straightened it.'

(388) No jeksh dyim. Wächeda'anma.

no hekš $\quad y-\operatorname{dim} \quad \emptyset$-wiče?-da?ay=ma

no now $3 \mathrm{~S}_{\mathrm{A}}$-say $3 \mathrm{~S}_{\mathrm{B}}$-big-old=IAM

“"Not now," she says, "Now it's big already.'

(389) Dyim njune'ep ñyumwatp, dyim, wo'kskä'da'apä' dyim.

y-dim nhune?e-pa ny-du:mu?-wat-p y-dim

$3 \mathrm{~S}_{\mathrm{A}}$-say how-FUT 2A:30-straight-do-FUT $3 \mathrm{~S}_{\mathrm{A}}-$ say

$\emptyset$-wo?ks-ki?da?a=pi? $\quad \mathrm{y}-\mathrm{dim}$

$3 \mathrm{~S}_{\mathrm{B}}-$ crooked-ADJZ=REL $3 \mathrm{~S}_{\mathrm{A}}-$ Say

"“How are you going to straighten that which is crooked?" she asks.'

(390) Eñdya du'umja'ap, dyim.

?eñdy ${ }^{y} \quad \emptyset-d u: m u ?-h-D a ?-p \quad y-d i m$

NEG $\quad 3 \mathrm{~S}_{\mathrm{B}}$-straight-LIG-CAUS-FUT $3 \mathrm{~S}_{\mathrm{A}}-$ say

“ "It won't straighten," she says.'

(391) Jepe' du'umja'ap jes wä'Đwä'Đna' dyim.

hepe? $\emptyset$-du:mu?-h-Da?-p hes $\emptyset$-wi? $\sim$ wi?n-na?

this $\quad 3 \mathrm{~S}_{\mathrm{B}}-$ straight-LIG-CAUS-FUT when $3 \mathrm{~S}_{\mathrm{B}}-$ DISTR little-still $\mathrm{y}-\mathrm{dim}$

$3 \mathrm{~S}_{\mathrm{A}}-$ say

“"This straightens when it is still little," she says.' 
(392) Jeksh dyim, bich, njyaam dyim.

hekš $y$-dim bič ny-ha:m y-dim

now $3 \mathrm{~S}_{\mathrm{A}}$-say $2 \mathrm{PRO} \quad 2 \mathrm{~A}: 3 \mathrm{O}-\mathrm{know} \quad 3 \mathrm{~S}_{\mathrm{A}}$-say

" Now," she says, "you know."

(393) Jeksh dyim, joye däk yojä' dyim, jepe' nje' dyim.

hekš y-dim hoye dik yoh-î y-dim hepe?

now $3 \mathrm{~S}_{\mathrm{A}}-$ say tomorrow $\mathrm{go}_{\mathrm{AUX}}$ pay-IMP $3 \mathrm{~S}_{\mathrm{A}}-$ say this

nhe? $\mathrm{y}-\mathrm{dim}$

3 PRO $3 \mathrm{~S}_{\mathrm{A}}-$ say

' "Now, tomorrow go pay for this!" she says.'

(394) Joye kdyikp kuyo'om.

hoye ky-dik-p kuy-olo-m

tomorrow $2 \mathrm{~S}_{\mathrm{B}}-$ go-FUT uncultivated.land-LOC-LOC

" "Tomorrow you're going to work (cultivate land).",

(395) Bweenu, pwe, wyokwoktet dyyuuñe'.

bueno pues y-wok wok-tet $\quad \mathrm{y}$-du:ñe?

good well 3A:3O-DISTR anger-burst 3S $\mathrm{S}_{\mathrm{A}}$-woman

'So, his wife is scolding him.'

(396) Ya ma' tzä'yam.

ya ma? $\emptyset-\phi i ? y=a m$

already PERF $3 \mathrm{~S}_{\mathrm{B}}$-stay=IAM

'That's where it ends.'

\section{REFERENCES}

Wichmann, Søren. 1996. Cuentos y colorados en popoluca de Texistepec. Copenhagen: Reitzels Forlag. 